\title{
The Structure of Titan's Atmosphere from Cassini Radio Occultations
}

\author{
Paul J. Schinder ${ }^{\mathrm{a}}$, \\ ${ }^{a}$ Center for Radiophysics and Space Research, Cornell University, Ithaca, NY \\ 14853 (U.S.A.) \\ F. Michael Flasar ${ }^{b}$ \\ ${ }^{\mathrm{b}}$ NASA Goddard Space Flight Center, Code 693, Greenbelt, MD 20770 (U.S.A.) \\ Essam A. Marouf ${ }^{\mathrm{c}}$ \\ ${ }^{\mathrm{c}}$ Department of Electrical Engineering, San Jose State University, San Jose, CA \\ 95192 (U.S.A.) \\ Richard G. French ${ }^{\mathrm{d}}$ \\ ${ }^{\mathrm{d}}$ Department of Astronomy, Wellesley College, Wellesley, MA 02481 (U.S.A.) \\ Colleen A. McGhee ${ }^{\mathrm{e}}$ \\ ${ }^{\mathrm{e}}$ Department of Astronomy, Wellesley College, Wellesley, MA 02481 (U.S.A.) \\ Arvydas J. Kliore ${ }^{f}$ \\ ${ }^{\mathrm{f}}$ Jet Propulsion Laboratory, Pasadena, CA \\ Nicole J. Rappaport ${ }^{\mathrm{g}}$
}


g Jet Propulsion Laboratory, Pasadena, CA

Elias Barbinis ${ }^{\mathrm{h}}$

${ }^{\mathrm{h}}$ Jet Propulsion Laboratory, Pasadena, CA

\section{Don Fleischman ${ }^{\mathrm{i}}$}

${ }^{\mathrm{i}}$ Jet Propulsion Laboratory, Pasadena, CA

Aseel Anabtawi ${ }^{\mathrm{j}}$

j Jet Propulsion Laboratory, Pasadena, CA

Number of pages: 38

Number of tables: 3

Number of figures: 12

Email addresses: schinder@astro.cornell.edu (Paul J. Schinder), f.m.flasar@nasa.gov (F. Michael Flasar), emarouf@email.sjsu.edu (Essam A. Marouf), rfrench@wellesley.edu (Richard G. French), cmcghee@firstclass.wellesley.edu (Colleen A. McGhee), akliore@jpl.nasa.gov (Arvydas J. Kliore), Nicole.J.Rappaport@jpl.nasa.gov (Nicole J. Rappaport), elias.barbinis@jpl.nasa.gov (Elias Barbinis), don.u.fleischman@jpl.nasa.gov (Don Fleischman), Aseel. Anabtawi@jpl.nasa.gov (Aseel Anabtawi). 
Proposed Running Head:

The Structure of Titan's Atmosphere

Please send Editorial Correspondence to:

Paul J. Schinder

1034 Karen St.

Boalsburg, PA 16827

Email: schinder@astro.cornell.edu

Phone: (814) 466-2150 


\section{ABSTRACT}

We present results from the two radio occultations of the Cassini spacecraft by Titan in 2006, which probed mid-southern latitudes. Three of the ingress and egress soundings occurred within a narrow latitude range, $31-34^{\circ} \mathrm{S}$ near the surface, and the fourth at $52.8^{\circ} \mathrm{S}$. Temperature - altitude profiles for all four occultation soundings are presented, and compared with the results of the Voyager 1 radio occultation (Lindal et al., 1983), the HASI instrument on the Huygens descent probe (Fulchignoni et al., 2005), and Cassini CIRS results (Flasar et al., 2005; Achterberg et al., 2008b). Sources of error in the retrieved temperature - altitude profiles are also discussed, and a major contribution is from spacecraft velocity errors in the reconstructed ephemeris. These can be reduced by using CIRS data at $300 \mathrm{~km}$ to make along-track adjustments of the spacecraft timing. The occultation soundings indicate that the temperatures just above the surface at $31-34^{\circ} \mathrm{S}$ are about $93 \mathrm{~K}$, while that at $53^{\circ} \mathrm{S}$ is about $1 \mathrm{~K}$ colder. At the tropopause, the temperatures at the lower latitudes are all about $70 \mathrm{~K}$, while the $53^{\circ} \mathrm{S}$ profile is again $1 \mathrm{~K}$ colder. The temperature lapse rate in the lowest $2 \mathrm{~km}$ for the two ingress (dawn) profiles at 31 and $33^{\circ} \mathrm{S}$ lie along a dry adiabat except within $\sim 200 \mathrm{~m}$ of the surface, where a small stable inversion occurs. This could be explained by turbulent mixing with low viscosity near the surface. The egress profile near $34^{\circ} \mathrm{S}$ shows a more complex structure in the lowest $2 \mathrm{~km}$, while the egress profile at $53^{\circ} \mathrm{S}$ is more stable.

Keywords: TITAN; ATMOSPHERES,STRUCTURE 


\section{Introduction}

Titan is unique among the moons of the solar system in having a thick, extended atmosphere, with a surface pressure exceeding 1 bar (1000 hPa). $\mathrm{N}_{2}$ is the dominant constituent, followed by $\mathrm{CH}_{4}$, which has a mole fraction of 0.015 in the stratosphere increasing at lower altitudes in the troposphere to several percent at the surface (Niemann et al., 2005, 2010). The most detailed measurements of temperatures in Titan's troposphere and middle atmosphere (stratosphere and mesosphere) have been made by instruments on the Cassini orbiter and Huygens probe. The Huygens Atmosphere Structure Instrument (HASI) obtained temperatures along the descent trajectory near $10^{\circ} \mathrm{S}$ (Fulchignoni et al., 2005). Between $1400 \mathrm{~km}$ and $160 \mathrm{~km}$, atmospheric mass density was calculated from accelerometer data and using a drag coefficient characterizing the probe. Application of the barometric law yielded pressure, and the ideal gas law provided temperature. Below $160 \mathrm{~km}$, with the parachutes deployed, temperature and pressure were directly measured by sensors. Vertical resolution was determined by the time sampling, and it was $20 \mathrm{~km}$ between $400 \mathrm{~km}$ and $1400 \mathrm{~km}$ altitude, decreasing to $1 \mathrm{~km}$ near 160 km (Fulchignoni et al., 2005). At lower levels it was finer, approximately 10 m just above the surface (Tokano et al., 2006).

In the thermal infrared, the Composite Infrared Spectrometer (CIRS) on the Cassini orbiter has mapped temperatures globally (Flasar et al., 2005; Achterberg et al., 2008b). In principle, it can retrieve temperatures between 300 mbar $(\sim 28 \mathrm{~km})$ in the upper troposphere (Flasar et al., 2004$)$ and $\sim 3 \mu$ bar $(\sim 470$

km, Achterberg et al., 2008b). To date, however, little has been reported on temperatures in the lower stratosphere between 100 and 10 mbar (50-100 km). 
This is because, in addition to collision-induced absorption from $\mathrm{N}_{2}-\mathrm{N}_{2}$ and $\mathrm{N}_{2}-\mathrm{CH}_{4}$ pairs, aerosols and condensates also contribute to the far-infrared opacity, and the latter have not been fully analyzed yet. Because of the lowpass filtering in the radiative transfer process and the distance of the spacecraft from Titan when limb-viewing observations are made, the vertical resolution in the CIRS temperature retrievals is typically a pressure scale height, e.g., $\sim 40 \mathrm{~km}$ in the middle atmosphere. Temperatures at higher altitudes in the mesosphere $(\sim 500 \mathrm{~km})$ have also been retrieved from solar and stellar occultations by the ultraviolet spectrometer on Cassini (e.g., Shemansky et al., 2005) and above $400 \mathrm{~km}$ from stellar occultations observed from Earth-based observatories (e.g., Hubbard et al., 1993; Sicardy et al., 2006).

Compared to the other Cassini orbiter experiments, radio occultations are the only means of obtaining temperatures in the lowest 1.5 scale heights of Titan's atmosphere, as well as the lower stratosphere at present. Moreover, the retrieved temperatures have much higher vertical resolution, which can, for example, resolve thin layers and waves with small vertical wavelengths. The technique, in which a radio signal from a spacecraft is refracted by the atmosphere of a target body before being recorded at a station on the Earth, can determine the structure of an atmosphere over several decades of pressure. It has been successfully used for many bodies in the solar system, including Jupiter and its satellites (Eshleman, 1975; Hinson et al., 1997), Saturn (Lindal et al., 1985), Uranus (Lindal et al., 1987), Neptune and Triton (Lindal, 1992), Mars (Cahoy et al., 2006), and Venus (Jenkins et al., 1994; Hinson and Jenkins, 1995). Prior to Cassini, the Voyager 1 spacecraft was occulted by Titan in 1979, early in Titan's northern spring (Lindal et al., 1983). The two radiooccultation soundings were both near the equator (Table 1). 
[Table 1 about here]

In 2006 (during Titan's northern winter), the second and third radio occultations of a spacecraft by Titan occurred. The Cassini spacecraft, in orbit around Saturn, was occulted twice by Titan, and Cassini obtained soundings by radio occultation at more southerly latitudes (Table 1) than Voyager. The first occultation occurred on orbit 22 during the T12 flyby on March 19. (Tn designates the sequence of the targeted Titan encounters. In order these are TA, TB, TC [when the Huygens probe descended into Titan's atmosphere], T3, T4, T5, etc.) The second occultation, T14 on orbit 24, occurred on May 20. The Cassini radio-occultation experiment differs in two important ways from that on Voyager (Kliore et al., 2004). First, for the first time, there are three frequencies: S-band $(2.3 \mathrm{GHz}), \mathrm{X}$-band $(8.4 \mathrm{GHz})$, and Ka-band (32 GHz); Voyager had only S and X. Second, the Cassini Ultra Stable Oscillator (USO) is a factor $\sim 25$ more stable than Voyager's. The latter permits sounding the neutral atmosphere higher than the $200 \mathrm{~km}$ achieved with the Voyager occultation soundings (Lindal et al., 1983). Since error in the bending angle (the angle between the initial ray direction as it enters the atmosphere and the final direction as it leaves) is directly related to error in the frequency, and the bending angle is smaller at high altitudes, a more stable frequency source with smaller errors gives us the capability to retrieve the temperature profile at higher altitudes.

Here we report the results of the analysis of the 2006 occultations. Two additional occultations at higher latitudes in both hemispheres occurred in 2007 during Cassini's primary mission. During the extended mission, one occultation at mid latitudes occurred in November 2008, and another ingress only occultation at high northern latitude in June, 2009. These will be the subject 
of a separate paper.

\section{The 2006 occultations}

\subsection{Procedure and Results}

During the T12 occultation, Cassini transmitted at three frequencies referenced to the on board USO (Kliore et al., 2004). The signal was collected using three antennas at the Deep Space Network station at Goldstone, California (DSS-14, 25, and 26), and two antennas at the DSN station at Madrid, Spain (DSS-55 and 63). Data were simultaneously recorded at the S (2.3 GHz),

$\mathrm{X}(8.4 \mathrm{GHz})$, and $\mathrm{Ka}(32 \mathrm{GHz})$ bands. The T14 occultation was also recorded at the three bands by the Madrid Deep Space Network station over the 70-m antenna DSS-63 (S and X) and the 34-m DSS-55 (X and Ka). During both occultations the boresight of the high-gain antenna on Cassini was continuously pointed at the predicted position of the virtual image of the Earth, that point on Titan where the refraction of the atmosphere will bend the ray so that it will intercept the Earth. At reception, the received signal from the spacecraft was "steered" by mixing with a reference frequency, which was computed by using a model Titan atmosphere and a code that predicts the received frequency of rays passing through that atmosphere, into a low frequency band that can be digitally recorded. The resultant mixed signal was then sampled at three different rates, 1,16 , and $50 \mathrm{kHz}$.

To determine the structure of the atmosphere of Titan with these data, we begin by Fourier analyzing the samples within a time window to determine the frequency at that time. Throughout this paper, we use the data sampled at 
$16 \mathrm{kHz}$, and use 4096 data points (.256 seconds) in a sliding window, with the window sliding 512 points (.032 seconds) between each frequency determination. Adding this to the reference frequency allows us to reconstruct the time series of the sky frequency, the original frequency of the signal as received at the DSN antenna. By averaging a series of those rays that don't enter Titan's atmosphere ("the baseline"), prior to the beginning of the actual sounding by the atmosphere (or after it, in the case of an egress sounding), and knowing the positions and relative velocity velocities of the spacecraft and DSN receiving station, we can determine the frequency at which the spacecraft transmitted in its rest frame. The fractional difference between the transmitted and the sky frequencies provides the initial ray direction, or, more precisely, the angle between the ray direction and the spacecraft velocity vector. Knowledge of the initial ray direction and final ray direction allows us to determine the refractivity profile of the atmosphere, as explained below.

We assume that the atmosphere of Titan is windless and spherically symmetric. Under these conditions, the Abel transform technique (Phinney and Anderson, 1968; Fjeldbo et al., 1971) can be used to retrieve the refractivity structure of the atmosphere. (The refractivity $N$ is related to the index of refraction $n$ by $N=10^{6}(n-1)$.) We can alternatively use ray tracing, a more general technique that assumes the atmosphere is axisymmetric and barotropic. Ray tracing provides additional useful information, such as the actual paths of the rays through the atmosphere, but at a much higher computational cost. However, the refractivity structure found by our ray tracing and Abel codes are identical for a spherically symmetric atmosphere, and the results presented below were obtained with our Abel code.

Figure 1 depicts the fractional power transmitted through Titan's atmosphere 
during the ingress and egress of T12 and T14. Atmospheric absorption, in addition to refractive defocusing, occurs at the $\mathrm{X}$ - and Ka bands. The signal was tracked all the way to the surface for $\mathrm{S}$ and $\mathrm{X}$ bands, while the Ka signal disappeared at about $10 \mathrm{~km}$ above the surface. The primary contribution to absorption is from pressure-induced absorption by $\mathrm{N}_{2}$ (Ho et al., 1968; Borysow and Frommhold, 1986; Lindal et al., 1985). A more detailed analysis of atmospheric absorption on Titan at radio wavelengths will be given elsewhere.

[Figure 1 about here.]

The Abel (or the ray-tracing) code derives, from the top down, a profile of the refractivity of the gas as a function of altitude. We obtain the number density by dividing by the refractivity per molecule, and the mass density by multiplying by the mean molecular weight of the gas. We assume the atmosphere of Titan is mostly $\mathrm{N}_{2}$, with a small admixture of $\mathrm{CH}_{4}$ at a level of $1.4 \times 10^{-2}$ by number (Niemann et al., 2005). The corresponding refractivity per molecule used for $\mathrm{N}_{2}$ is $1.1 \times 10^{-17} \mathrm{~cm}^{3}$ per molecule, and that for $\mathrm{CH}_{4}$ is $1.63 \times 10^{-17} \mathrm{~cm}^{3}$ (Achtermann et al., 1991; Orcutt and Cole, 1967; Essen and Froome, 1951). The equation of hydrostatic equilibrium is integrated downwards from a chosen starting altitude and temperature. We use the Bender equation of state, which takes into account the departure of the equation of state from a perfect gas at the low temperatures and high pressures characteristic of the lower troposphere and conveniently permits the treatment of gas mixtures (Bender, 1971, 1973; Bühner et al., 1981).

[Figure 2 about here.]

Figure 2 shows the latitude and longitude of the ray periapses as they descend toward (or ascend from, during egress) the surface of Titan. The soundings 
usually cover several degrees of latitude and longitude as the virtual image of the spacecraft as seen from the earth descends toward (ascends from) the surface. Figure 3 shows the temperature-altitude profile for the region between 0 and $300 \mathrm{~km}$ for the T12 and T14 soundings. The integration was started as near to $300 \mathrm{~km}$ as possible at $185 \mathrm{~K}$; at that altitude the CIRS profiles we use to obtain a starting value have an error of about $\pm 1 \mathrm{~K}$ (Achterberg et al., 2008b). Appendix A contains tables that give the values we obtain for the pressure, temperature, and refractivity at selected altitude levels for all four soundings. As described below, we have adjusted the spacecraft ephemeris so that the temperature profile in the stratosphere closely matches that obtained by CIRS observations.

[Figure 3 about here.]

The profiles given in the tables and the figures have been retrieved from $16 \mathrm{kHz}$ $\mathrm{X}$-band data. We have found that profiles in the neutral atmosphere below 300 $\mathrm{km}$ retrieved from $\mathrm{X}$ and Ka-band data are little affected by refraction from electrons in Titan's weak ionosphere. The S-band data, however, are noticeably affected by the presence of electrons. It is well established that this effect can be removed by using two frequencies. For example, the refractivity derived from $\mathrm{S}$ band data $n_{S}$ is assumed to consist of two parts, $n_{0}$ independent of frequency and $n_{i}(f)$ a function of frequency, and similarly for the $\mathrm{X}$ band refractivity $n_{X}$. It follows that $n_{0}=\left(n_{S}-\left[n_{i}\left(f_{S}\right) / n_{i}\left(f_{X}\right)\right] n_{X}\right) /\left(1-\left[n_{i}\left(f_{S}\right) / n_{i}\left(f_{X}\right)\right]\right)$ (Schunk and Nagy, 2000). Since $n_{i} \propto f^{-2}$ for free electrons, we can use the $\mathrm{X}$ and $\mathrm{S}$ band, or any other two bands, to extract the refractivity $n_{0}$ of the neutral gas alone. We have found that the retrieved temperatures using the $\mathrm{X}$ - or Ka-band (above $10 \mathrm{~km}$ ) alone are virtually identical to the profiles obtained by combining $\mathrm{X}$ and $\mathrm{S}$ bands in the altitude range we present here. 


\subsubsection{Diffraction}

Because we use geometric optics in the retrievals, diffraction limits the vertical resolution of structure in the atmosphere to the diameter of the first Fresnel zone (Fjeldbo and Eshleman, 1969). In the stratosphere, the diameter is close to the free-space value, $2 \sqrt{\lambda D}$, where $\lambda$ is the wavelength of the radio signal and $D$ the distance of Cassini from Titan's occulting limb. For $\mathrm{X}$ band, this is typically $\sim 1 \mathrm{~km}$ for the T12 and T14 occultations. Differential refraction in a

neutral atmosphere reduces the vertical scale by a factor $\sim 1 / \sqrt{(1+D \alpha / H)}$, where $H$ is the pressure scale height and $\alpha$ is the bending angle between the incoming and outgoing ray asymptotes (Haugstad, 1978). In the lower troposphere $D \alpha / H>>1$, and the vertical diameter of the first Fresnel zone is $\sim 2 \sqrt{\lambda H / \alpha} \sim 300 \mathrm{~m}$ for $\mathrm{X}$ band, independent of $D$.

Titan has a solid surface, and the surface can cause diffraction in the last $\sim 300 \mathrm{~m}$ of the temperature profile. Since diffraction would cause a deviation from the geometric optics solution, the Abel transform (or ray tracing), which assumes geometric optics, might misinterpret diffraction as a temperature structure right at the surface. Since we use exclusively the frequency record and not the power record, we are sensitive only to the deviation in the actual recorded frequency from the geometric optics prediction. It should be noted that there is no convincing signal in either the power record or the frequency record that diffraction is occurring at the surface, but this may be due to the fact that the power is also dropping rapidly at the surface due to refractive defocusing (for the $\mathrm{X}$ and Ka bands, pressure induced absorption by $\mathrm{N}_{2}$ is also a factor). Anything, not just diffraction, that could cause a deviation from the geometric optics solution in a spherically symmetric atmosphere (such as we are assuming for the Abel transform) could cause deviations of 
the computed temperature profile from the actual temperature profile. These include actual atmospheric structure that is not spherically symmetric, both large scale zonal and longitudinal deviations from spherical symmetry and small scale deviations (waves, etc.). The reverse is also true: structure that actually exists in the atmosphere near the surface (local surface inversions, for example, or deviations caused by terrain) may be misinterpreted as the effects of diffraction.

French and Gierasch (1976) show that the effects of diffraction are greatly reduced in the presence of a thick atmosphere, such as found on Titan. One way to remove the effects of diffraction from the recorded data and therefore be able to distinguish between real surface effects and diffraction is backpropagation (Karayel and Hinson, 1997; Marouf et al., 1986). Backpropagation in effect synthetically shifts the trajectory of the spacecraft to one closer to the target body so that the Fresnel zone becomes arbitrarily small. In this case, backpropagation must be done taking into account the presence of a thick atmosphere between the DSN station recording the frequency data and the spacecraft. We have devised a procedure to do this, but the details and the results of both backpropagation and the results of forward calculations using a simplified geometry will be held for a future paper to allow a full discussion of the problem. The results we present here are not backpropagated and the vertical resolution is diffraction limited.

\subsection{Sources of error}

In this section, we will discuss some of the sources of error that affect the profiles, particularly those that affect the profiles preferentially above $70 \mathrm{~km}$, 
and how we have corrected for them when possible.

\subsubsection{Hydrostatic equilibrium starting point}

First, the computation of the temperature and pressure profiles from the refractivity profiles starts at a given altitude with an arbitrarily chosen temperature.

[Figure 4 about here.]

Figure 4 shows the effect of starting the hydrostatic equilibrium calculation at $300 \mathrm{~km}$ at three different temperatures, 175, 185, and $195 \mathrm{~K}$, for T14 egress using $\mathrm{X}$ band data. Note that the shapes of the profiles remain the same, and the divergence decreases (approximately as $1 / P$, see Lipa and Tyler, 1979) as we descend, becoming negligible at about $150 \mathrm{~km}$. Figure 4 also shows the effect of starting the hydrostatic equilibrium calculation at 148 $\mathrm{km}$ at $172.5 \mathrm{~K}$, approximately the same as the first direct measurement by the HASI instrument on Huygens, again for T14 egress. Both profiles agree well, although the radio occultation profile does not show the abundant small scale structure seen in the Huygens profile. This is not surprising, because the Huygens in situ measurements detect local structure, whereas the occultation measurements detect an average along the ray path in a shell of thickness comparable to the vertical scale of the structure being resolved (provided it is larger than the Fresnel scale). This is roughly the geometric mean of Titan's radius $\left(R_{T}=2575 \mathrm{~km}\right)$ and the vertical scale. In Figure 4 the occultation retrievals would effectively average structure observed by Huygens on 10-km scales over a distance $\sim \sqrt{10 \times 2575} \approx 160 \mathrm{~km}$. If the horizontal scale of the small-scale structure measured by Huygens is much smaller, then it will be 
smoothed out.

In order to minimize the arbitrary starting point as a possible source of error in our profiles, we use CIRS profiles (Achterberg et al., 2008b) to set the temperature at $185 \mathrm{~K}$ at an altitude of $300 \mathrm{~km}$. At that altitude the CIRS retrievals have an error (from instrument noise) of approximately $\pm 1 \mathrm{~K}$.

\subsubsection{Baseline}

The start of every ingress occultation and end of every egress occultation includes a baseline, a period of time in which the signal from the spacecraft is tracked in "free space". This is done so that the spacecraft USO frequency in its rest frame can be determined from the data, to enable subsequent analysis. However, there are choices to be made in how to handle the baseline, and what to do with the inevitable deviations from the ideal of transmission in a vacuum.

The choice begins with an examination of the Doppler residual frequency, the difference between the actual earth received frequency and the earth received frequency predicted if the path between the spacecraft and the DSN antenna were in vacuum. For this purpose, the spacecraft oscillator frequency is determined from the first point in the time series of earth received frequencies vs. time.

[Figure 5 about here.]

Figure 5 shows an example for the T14 ingress occultation. There are several features to note. First, the Doppler residuals don't lie exactly on zero, as they would if both the receiver and transmitter are in vacuum. Instead, they show 
1) a subtle linear trend away from zero, 2) subtle small scale structure, and 3) a narrow spread in frequency due to thermal noise. Of course, the path from spacecraft to receiver isn't entirely in vacuum (the signal passes through the Earth's ionosphere and atmosphere, for example), so deviations from the ideal should be expected.

We next choose an interval in time. We usually try to choose an interval as long as possible that doesn't have obvious problems (excess curvature, discontinuities, etc.). We also need to avoid the target's ionosphere, if any. A baseline of more than $200 \mathrm{~s}$ is preferred, although not always possible. In the case shown in Fig. 5, we choose the interval 48000-48400 s. For some soundings, such as T12 ingress, the baseline is short for reasons beyond our control, and we have to accept the greater (mostly stratospheric) uncertainty that results. The errors caused by any sort of frequency uncertainty are always larger where the density and the bending angle are smaller, so they affect high altitudes the most.

Next, we numerically integrate the Doppler residuals to derive a phase, and then fit the phase with a cubic polynomial using a linear least squares fit. The derivative of this cubic gives us a quadratic fit to the residual frequency. We then determine the spacecraft oscillator frequency from this fit. Ordinarily we then remove the linear drift using the linear part of the fit, although occasionally the quadratic part is big enough so that it must be removed as well. In this way we get as close as possible to the ideal of the Doppler residuals being zero outside the atmosphere.

However, the baseline and what we do with the baseline is a subjective choice to some degree, and that choice and how we correct the data will affect the 
subsequent temperature - altitude profile. In order to minimize the effects of both the baseline choice and the spacecraft ephemeris errors which we describe next (which also cause errors in frequency), we choose to use CIRS profiles (Achterberg et al., 2008b) and adjust the ephemeris by introducing a timing offset between the time a given ray leave the spacecraft and the time at which we query the ephemeris for the spacecraft's position and velocity for that ray. This allows us to bring our stratospheric temperature - pressure profile into agreement with the CIRS profiles, while eliminating both the systematic errors caused by the choice of the hydrostatic equilibrium starting point and the choice of the baseline, leaving only random errors.

\subsubsection{Ephemeris errors}

The ephemerides provided by JPL come in the form of binary kernels which contain fits to the actual trajectory of the spacecraft, Saturn, and Titan for a certain time interval. The errors in both the position and the velocity of the spacecraft change with time along the trajectory. The position errors for the "reconstructed" kernels (kernels which contain the past trajectory of the spacecraft based on the best available tracking data) are on the order tens of meters near Titan. The velocity errors are on the order of a few mm/s.

We have equipped our code with the ability to do an along-track "time shift"; in other words, we query the ephemeris at time $t+\Delta t$ and use that state vector as if it were the correct one for time $t$. We perform this shift only for the spacecraft state vector, and not for Saturn, Titan, or the DSN antenna. This is a simple, one parameter attempt to estimate the effect of ephemeris

errors. More elaborate schemes can be envisioned (including, for example, 
using information from the atmospheric occultation to improve the ephemeris), but are much harder to implement.

[Figure 6 about here.]

Figure 6 shows the temperature profiles obtained for various values of $\Delta t$. The effects of even a few millisecond time shifts are quite apparent above $70 \mathrm{~km}$, with large temperature differences above $150 \mathrm{~km}$. The rejoining of the profiles at $300 \mathrm{~km}$ at $185 \mathrm{~K}$ is an artifact of the fact that the hydrostatic equilibrium calculation was started there.

Using a time shift means that both the position and the velocity of the spacecraft change from the nominal value. It turns out that the velocity change is the controlling factor. We have tested this by suppressing the change in either the position or the velocity caused by the time shift. While the shift in position of a few tens of meters causes only a very small $(<1 \mathrm{~K})$ temperature shift at high altitudes, the shift in velocity of $\sim 1 \mathrm{~mm} \mathrm{~s}^{-1}$ causes almost all of the shift shown in Fig. 6.

[Figure 7 about here.]

We have obtained from the Cassini Navigation (NAV) team a set of covariance matrices describing the error in position and velocity of the reconstructed SP (Spacecraft-Planetary) kernel from JPL's Navigation and Ancillary Information Facility (NAIF) for both of these occultations. With this, we are able to make a more realistic appraisal of the effect of ephemeris errors on the stratospheric temperature profile.

Shifting the velocity affects the temperature profile in two ways. First, the spacecraft USO frequency is determined from the sky frequencies recorded on 
the ground. The relation between the spacecraft oscillator frequency $f_{s}$ and the sky frequency $f_{a}$ in the rest frame of the DSN antenna is

$$
\frac{f_{a}}{f_{s}}=\frac{1}{1-\left(\mathbf{v}_{s} \cdot \hat{\mathbf{n}}\right) / c}
$$

to first order in $v / c$, where $\hat{\mathbf{n}}$ is the unit vector along the initial direction of the ray starting at the spacecraft and ending at the ground antenna, $\mathbf{v}_{s}$ is the spacecraft velocity, and $c$ the speed of light. As described above, we essentially average this equation over the baseline to get the constant spacecraft frequency $f_{s}$. So if the ephemeris returns a velocity of $\mathbf{v}+\delta \mathbf{v}$ when the spacecraft velocity is actually $\mathbf{v}$, the spacecraft oscillator frequency will be slightly in error by an amount

$$
f_{s}=f_{s_{0}}-<f_{a}(\delta \mathbf{v} \cdot \hat{\mathbf{n}}) / c>
$$

where $f_{s_{0}}$ is the actual spacecraft frequency and the angle brackets denote the averaging procedure.

Once the spacecraft oscillator frequency is obtained, we use it and the recorded ground frequencies to find the angle between the initial ray direction and the spacecraft velocity vector. The angle can easily be found from

$$
\frac{\mathbf{v}_{s} \cdot \hat{\mathbf{n}}}{c}=\frac{f_{a}-f_{s}}{f_{a}}
$$

The ray then must be bent by just the right amount to arrive at the DSN antenna with the measured frequency. This means that if the ray direction is slightly off from its actual direction due to ephemeris errors, the structure of the atmosphere computed by using the ray must change slightly to bend it in the right direction. This has a direct effect on the refractivity profile and an 
indirect effect on the temperature profile.

To begin, we choose a fixed direction at random. We then multiply that direction by a changing magnitude calculated from the covariance matrix. Because the covariance matrix changes with time, changing the magnitude of the velocity error gives a more realistic appraisal of the error caused by the ephemeris. The fixed direction for the velocity error (but not for the velocity itself) isn't as realistic, but the actual change in direction of the total velocity is controlled by celestial mechanics, and a fixed direction for the error vector is a more reasonable approximation (for error analysis only) for the short duration of an atmospheric occultation than to allow, say, the direction of the error component to randomly change with time.

The covariance matrices are $1 \sigma$ errors, so we performed a Monte Carlo estimation of the errors in the temperature - altitude profile by performing 100 cases each of $3 \sigma$ velocity errors. We again started the hydrostatic equilibrium integration at $300 \mathrm{~km}$ at $185 \mathrm{~K}$. Figure 7 shows the resulting temperature profiles for T14 egress in $\mathrm{X}$ band using $16 \mathrm{kHz}$ data. With $3 \sigma$, the maximum error is around $20 \mathrm{~K}$. Once again, the joining of the profiles at $300 \mathrm{~km}$ is an artifact of the initial conditions.

The $1 \sigma$ velocity errors provided by the NAV team for the T14 reconstructed SP kernel were 2 - $4 \mathrm{~mm} \mathrm{~s}^{-1}$ during the course of the occultation, while for T12 the errors were $0.5-1 \mathrm{~mm} \mathrm{~s}^{-1}$. Note that an error $\sim 1 \mathrm{~mm} \mathrm{~s}^{-1}$ corresponds to an additional fractional frequency shift of $0.1 \mathrm{~cm} \mathrm{~s}^{-1} / 3 \times 10^{10} \mathrm{~cm} \mathrm{~s}^{-1} \sim$ $3 \times 10^{-12}$, or roughly $25 \mathrm{mHz}$ for $\mathrm{X}$ band. In the lower stratosphere and troposphere the fractional shift in frequency from refraction is much larger, $\sim 10^{-7}$, so the effect of the velocity error damps out in this region, as Fig. 7 shows. 
Timing errors like the ones we discussed above are possible if the clock that timestamps the recordings in the radio science receivers are not in sync with the ephemeris time in the SP kernel. This may be possible, but the computers providing the timestamps for the radio science data files are kept in sync with a local highly accurate time reference. Our most likely source of this class of error for determining the stratospheric temperature profile is timing errors in the ephemeris itself.

[Figure 8 about here.]

We have attempted to correct for the errors introduced by the velocity shifts by making use of retrieved CIRS temperatures near $300 \mathrm{~km}$ (Achterberg et al., 2008b). To make the approach tractable, we do not alter the spacecraft velocities in the ephemeris, but instead introduce a single time shift in the ingress and egress portions of each occultation. We adjust the time shifts until the difference between our profiles and the CIRS profiles are small, bringing our stratospheric temperature - pressure profiles into close agreement with the profiles from CIRS. Figure 8 illustrates the quality of the fitted profiles. It is possible with along-track shifts within the quoted errors of the spacecraft ephemeris to align the RSS temperature profiles with the CIRS profiles to good accuracy.

The CIRS temperature profiles used are zonal averages based on a combination of limb and nadir observations from July 2004 to May 2007 (Achterberg et al., 2008a,b). The limb profiles are limited and sample longitude poorly, but pressures 0.2 to 5 mbar are dominated by nadir-sounding observations, which sampled longitude very well and typically consist of averages of $\sim 100$ or more retrieved temperature profiles within $5^{\circ}$-latitude bins. This pressure 
range dominates that of the fitting procedure illustrated in Fig. 8, and the errors in temperature over these pressures from the CIRS instrument noise are approximately $0.5 \mathrm{~K}$ for a single retrieved profile. When zonally averaged, this reduces by a factor of 10 or more. The radio-occultation retrievals are not zonal averages, but sounded over a narrow range of longitudes (Fig. 2). The CIRS temperatures near 1 mbar, however, indicate small variations around latitude circles at the latitudes of the occultation soundings, with a standard deviation of $<0.6 \mathrm{~K}$. Temporal variations in the CIRS profiles over the three-year period also appear to be small, with seasonal variations becoming apparent in 2008-2009. This is understandable, because the earlier observations shortly after the northern winter solstice, when there is an extremum in the solar declination.

\subsubsection{Random noise in the frequency}

Figure 5 shows a spread of frequencies around Doppler zero, in this case with a width of $\sim 40 \mathrm{mHz}$. The cause of this random error is thermal noise in the system.

To a certain extent the size of this random component is under our control. Fourier transforming more data for each frequency point improves the signal to noise ratio, but including too much data creates artifacts that can affect the profiles.

To estimate the effect that the random noise has on our profiles, we add white noise to the frequency record with the same $\sim 40 \mathrm{mHz}$ width, effectively doubling the noise, and do 100 trials. 
[Figure 9 about here.]

The results are shown in Fig. 9. For T14, the errors caused by white noise are roughly half as large as the errors caused by ephemeris errors (see also Fig. $3)$.

\subsubsection{Other sources of error}

Other sources of error include the assumed composition of the atmosphere and the value of the refractivity per molecule that we use. If some other constituent of the Titan atmosphere that we didn't consider had an unusually large re-

fractivity per molecule, then we would overestimate the density (the number of molecules required to obtain the observed refractivity), and this would affect our temperature profiles. However, the temperature profiles determined by radio occultation and other techniques agree well in the lower atmosphere, so errors of this sort are probably not large. As we showed above, the starting value of the hydrostatic equilibrium calculation can cause substantial errors in the stratosphere. However, the structure of the atmosphere at $300 \mathrm{~km}$ is well determined by other techniques, and we are using one of them to set the temperature at $300 \mathrm{~km}$, so in practice this should not be a significant source of error.

\section{Discussion}

The left panels $(a, c, e)$ of Fig. 10 display the T12 and T14 profiles together. The most notable feature is how similar they are, although differences do exist. Above $100 \mathrm{~km}$, T14 egress is roughly $2.5 \mathrm{~K}$ warmer than the profiles at T14 
ingress and T12 ingress, even though all are at nearly the same latitude. This is within the 1- $\sigma$ errors of these retrievals from thermal noise at these altitudes (Fig. 3). Nadir sounding by CIRS (Achterberg et al., 2008a) indicates little zonal variation near 1 mbar at these latitudes. However, it is possible that the fits to the CIRS profiles in the upper stratosphere by simply shifting the timing of the spacecraft ephemeris has introduced some residual systematic error. The right panels $(b, d, f)$ compare the T14 retrievals with those from Voyager radio occultations and HASI. The Voyager profiles, although equatorial and at a different season, are nonetheless close to those from T12 and T14. The HASI profile is consistently warmer in the stratosphere, although this may in part be attributable to adjusting the spacecraft ephemeris and boundary condition to the CIRS profiles in inverting the radio-occultation data. There is a discrepancy between HASI and the CIRS retrievals at low latitudes that is not understood. The HASI profiles are $\sim 10 \mathrm{~K}$ warmer in the upper troposphere, and calculated synthetic spectra using these profiles produce infrared spectra that have radiances near $7 \mu \mathrm{m}$ that are much too high compared to CIRS observations (Coustenis et al., 2005; Ferri et al., 2006; Flasar and Achterberg, 2009).

The three profiles at $32-34^{\circ} \mathrm{S}$ are virtually identical in the tropopause region (panel $c, 30-55 \mathrm{~km}$ ). The profile at the higher latitude, $53^{\circ} \mathrm{S}$, is approximately $0.8 \mathrm{~K}$ cooler near $40 \mathrm{~km}$. The $32-34^{\circ} \mathrm{S}$ profiles very close to those from HASI at these altitudes, but up to $0.4-0.8 \mathrm{~K}$ cooler than those from Voyager radio occultations (panel $d)$.

Closer to the surface (panel $e$ ), the profiles at $32-34^{\circ} \mathrm{S}$ are close to dry adiabatic in the lowest $2 \mathrm{~km}$, and gradually becoming more stable above that. This can be seen in Fig. 11, which depicts the vertical gradients of temperature 
in the lowest $10 \mathrm{~km}$. In the T12 ingress and T14 ingress profiles, there is an indication of more stable behavior below the adiabatic layers just above the surface. Both ingress soundings occurred near the dawn terminator, and this stability may reflect surface cooling overnight. A stable nocturnal inversion would not be radiatively driven, but more likely induced by turbulent mixing with low effective viscosity (see Appendix B). An adiabatic layer extending up to $2 \mathrm{~km}$ is more or less consistent with the Voyager results of Lindal et al. (1983), but it is much greater than the $300 \mathrm{~m}$ height inferred from the HASI data near $10^{\circ} \mathrm{S}$ (Tokano et al., 2006). However, one should bear in mind that the HASI measurements are local, whereas the radio occultation temperature at a given altitude is really an average of the atmospheric structure over the ray path, as discussed earlier in section 2.2.1.

The $32-34^{\circ} \mathrm{S}$ temperatures and those from HASI and the Voyager occultation are virtually identical at the surface (panel $f$ ). At higher altitudes, the HASI profile is relatively warmer, about $0.7 \mathrm{~K}$ at $6 \mathrm{~km}$. The Voyager profiles are not as warm, and lie closer to the Cassini radio-occultation temperatures. The profile at $53^{\circ} \mathrm{S}$, near the evening terminator, is more stably stratified below $3 \mathrm{~km}$ than the lower-latitude Cassini profiles. At $0 \mathrm{~km}$, relative to a $2575-\mathrm{km}$ sphere, it is $0.9 \mathrm{~K}$ cooler than the other three (panel $e$ ).

Over much of the region depicted in Fig. 11, the temperature gradients exhibit a fine-scale "spiky" structure. This fine structure may reflect ray crossing attributable to small-scale vertical variations in the refractivity. French and Lovelace (1983) have examined the distortion that a sinusoidal variation in refractivity of sufficiently large amplitude can produce in temperature profiles retrieved from stellar occultations using geometric optics. Appendix C briefly describes the effect for for a sinusoidal variation added to an isothermal at- 
mosphere. A real atmosphere will probably have a spectrum of such waves, and a more complex structure. We have made a preliminary study using the backpropagation procedure mentioned in section 2.1.1, and we find that the spikes have smaller amplitudes as the observer's trajectory is synthetically shifted toward the occulting limb, which suggests that ray crossing is indeed occurring. Note that the spiky behavior in Fig. 11 is largely absent from the adiabatic layers. This may be because the more rapid vertical mixing in these layers has smoothed out the small perturbations. A sharp feature at $1 \mathrm{~km}$ altitude appears to be "embedded" within the adiabatic layer of the T14 egress profile, which is near the evening terminator. It might represent a thin stable layer, but this remains a speculation.

All the radio-occultation profiles displayed correspond to a uniform distribution of the $\mathrm{CH}_{4}$ mole fraction at 0.015 . To check the sensitivity of the profiles to variable $\mathrm{CH}_{4}$ concentrations, temperatures were retrieved for the T14 ingress $\left(33^{\circ} \mathrm{S}\right)$ assuming the vertical variation obtained by the Huygens Gas Chromatograph Mass Spectrometer (GCMS): being $\approx 0.015$ down to $40 \mathrm{~km}$, increasing to 0.024 at $20 \mathrm{~km}$, then increasing more rapidly with decreasing altitude to 0.057 near the surface (Niemann et al., 2010). The effect, illustrated in panel $f$ (blue dotted curve), is small. The differences are largest in the lowest $10 \mathrm{~km}$, and the profile with variable $\mathrm{CH}_{4}$ is about $0.6 \mathrm{~K}$ warmer at $5 \mathrm{~km}$ altitude. The retrieval with the $\mathrm{GCMS} \mathrm{CH}_{4}$ distribution in panel $f$ is nearly coincident with the HASI profile.

For a uniform $\mathrm{CH}_{4}$ distribution, saturation will most easily occur in the tropopause region, because the temperatures there are lowest. For the temperature profiles near $30^{\circ} \mathrm{S}$ with 0.015 uniform $\mathrm{CH}_{4}$, the relative humidity over $\mathrm{CH}_{4}$ ice is $<87 \%$; at $53^{\circ} \mathrm{S}$ it is $<96 \%$. Condensed $\mathrm{CH}_{4}$ and $\mathrm{N}_{2}$ are partially 
miscible in the liquid and probably solid phases (Omar et al., 1962). If $\mathrm{CH}_{4}$ and $\mathrm{N}_{2}$ are mixed in the ice phase, then the saturation vapor pressure of $\mathrm{CH}_{4}$ would be less than over its pure solid phase and saturation might occur in the tropopause region. However, there are no available data on the properties of the vapor-solid phase equilibrium of $\mathrm{CH}_{4}-\mathrm{N}_{2}$ mixtures, and what occurs on Titan is controversial. Tokano et al. (2006) have argued that the HASI temperature and GCMS $\mathrm{CH}_{4}$ gas profiles above the lowest few $\mathrm{km}$ are consistent with saturation over liquid $\mathrm{CH}_{4}-\mathrm{N}_{2}$ mixtures, but above the freezing level near $15 \mathrm{~km}, \mathrm{CH}_{4}$ vapor seems to be governed by the saturation pressure over $\mathrm{CH}_{4}$ ice.

[Figure 10 about here.]

[Figure 11 about here.]

\section{Acknowledgments}

We thank the JPL Radio Science Systems group and the personnel of the NASA/JPL Deep Space Network for their key roles in the successful acquisition of the radio science data.

\section{Appendix A: Titan Tables}

This appendix presents two tables, Table 2 and Table 3. These tables contain numerical values for the temperature, pressure, and refractivity we obtain for the atmosphere of Titan for the occultations discussed in the main text at specific altitudes in the atmosphere. These numerical values at specific altitudes are obtained by linear interpolation from a smoothed version of our 
computed results, which are not uniformly spaced in altitude. The smoothing was done to reduce interpolation artifacts on the coarse altitude grid presented in these tables.

[Table 2 about here]

[Table 3 about here]

\section{Appendix B: Nocturnal Inversion}

The three soundings near $30^{\circ} \mathrm{S}$ are close to dry adiabatic over much of the lowest $2 \mathrm{~km}$. This is particularly evident in the T12 and T14 ingress soundings (Fig. 11). Moreover, both exhibit a stable inversion in the lowest $\sim 200 \mathrm{~m}$. The local solar time of both soundings is shortly before 5 A.M. This suggests that cooling of the surface drives the inversion. One can readily show that radiative cooling of the atmosphere just above the surface is too inefficient to establish a stable inversion on diurnal timescales. For a grey absorption the relaxation time $\tau(m)$ to a sinusoidal perturbation of vertical wavenumber $m$ is given by (Goody and Yung (1989), Ch. 10)

$$
\frac{1}{\tau(m)}=\frac{4 \pi k_{v}}{\rho C_{p}} \frac{d B}{d T}\left[1-\frac{k_{v}}{m} \tan ^{-1}\left(\frac{m}{k_{v}}\right)\right]
$$

where $\rho$ is atmospheric mass density, $C_{p}$ is the specific heat at constant pressure, $k_{v}$ is the volume absorption coefficient, with units of $1 /$ length, $T$ is temperature, and $B=\pi^{-1} \sigma_{B} T^{4}$ is the Planck function integrated over all frequencies; $\sigma_{B}=5.67 \times 10^{-8} W^{-2} s^{-1} K^{-4}$ is the Stefan-Boltzmann constant. Assuming radiative equilibrium and using the temperatures retrieved from the Voyager radio-occultation soundings, Samuelson (1983) estimated that $k_{v} / \rho \sim 7.6 \times 10^{-5} \mathrm{~m}^{2} \mathrm{~kg}^{-1}$ just above the surface. With $1 / \mathrm{m} \sim 200 \mathrm{~m}$, 
$\tau(m) \sim 263$ days, much longer than Titan's 15.95-day diurnal period.

However, a modest amount of turbulent mixing just above the surface, can account for the stable inversion over diurnal time scales. An eddy viscosity can be estimated as

$$
D=h^{2} / t
$$

where $h$ is the vertical scale of the boundary layer, $\sim 200 \mathrm{~m}$, and $t$ is the time scale of the diurnal cycle, $15.95 \mathrm{~d} / 2 \pi \approx 2.2 \times 10^{5} \mathrm{~s}$. This yields $D \sim 0.1 \mathrm{~m}^{2} \mathrm{~s}^{-1}$, which is comparable to the global tropospheric value estimated by Flasar et al. (1981). For a 300-m boundary layer, Tokano (Tokano et al., 2006) estimated a value of $D$ that was an order of magnitude smaller. However he related the eddy viscosity to the height of the Ekman layer where the surface winds are nearly geostrophic, which is a factor of $\pi$ larger than the vertical scale of the layer. Hence, for a given layer height and time scale, his estimate of $D$ gave a value $\pi^{2}$ smaller, which accounts for the difference. Note that the above estimate of $D$ pertains to the stable inversion layer adjacent to the surface. The adiabatic layers above this likely have larger values, at least during daylight hours.

\section{Appendix C: The Effects of Ray Crossing from Sinusoidal Structure in an Isothermal Atmosphere}

The mean atmospheric structure typically has vertical profiles of density and refractivity that increase with decreasing altitude, on a scale comparable to the pressure scale height, $H$. Consider the simple case of an isothermal atmosphere 
with constant $H$. When the bending angle, $\alpha$, is small,

$$
\alpha \approx \sqrt{\frac{2 \pi R}{H}} N_{0} \exp \left(-\frac{z}{H}\right)
$$

where $N_{0}$ is the refractivity at $z=0$, and $R$ is the radius from the center of the occulting body to the ray periapsis point (Baum and Code, 1953). The dominant variation in the bending angle with altitude is from the exponential term, i.e., the bending angle to first order increases linearly with the refractivity.

Now superpose a sinusoidally varying component to the exponentially varying refractivity, with fractional amplitude $\epsilon$ and vertical wavenumber $m$. The bending angle now does not necessarily increase monotonically with decreasing $z$. At altitudes where the perturbation increases with decreasing $z, \alpha$ will be larger than in the absence of the perturbation; where the perturbation decreases with decreasing $z, \alpha$ will be smaller. This leads to the possibility of ray crossing. French and Lovelace (1983) found that crossing could occur in principle when

$$
(m H)^{3 / 2} \epsilon>1
$$

Ray crossing becomes more likely for perturbations with larger amplitudes and smaller vertical scales.

French and Lovelace (1983) were concerned with the ambiguities that can arise when inverting light curves from stellar occultations. When ray crossing occurred, their model light curves exhibited intense spikes. To illustrate the effect of ray crossing on retrieved temperature profiles when using an Abel inversion, we have used their algorithm and calculated the light curves in an exponential atmosphere for several cases with sinusoidal perturbations of different ampli- 
tudes. The light curves generated by ray tracing in the model atmospheres were inverted by means of an Abel transform. Figure 12 illustrates the input sinusoidal and retrieved temperatures, and also the vertical gradients of the retrieved temperatures. For low amplitudes, there is no ray crossing, and the Abel inversions faithfully reproduce the effects of the perturbed atmosphere structure. However, when the fractional amplitude becomes 0.03 or larger, distortions become apparent. At $\epsilon=0.1, d T / d z$ exhibits a spiky structure that is reminiscent of that in Fig. 11. The vertical wavelength in Fig. 12 is $H / 2 \approx 10$ km, whereas the structure in Fig. 11 is smaller, for example, 300-400 $\mathrm{m}$ for the strongest spikes in the T12 ingress panel, about 30 times smaller. The above scaling suggests that the spiky structure observed in Titan's lower atmosphere

may correspond to fractional amplitudes of order $0.1 / 30^{3 / 2}=6 \times 10^{-4}$, or 0.06 K. It should be noted that this corresponds to the average structure over a ray path distance comparable to the geometric mean of the vertical wavelength and Titan's radius, several tens of kilometers. Nonetheless, small perturbations can have a noticeable effect on the Abel inversions, if the vertical scales are small enough.

[Figure 12 about here.]

\section{References}

Achterberg, R. K., Conrath, B. J., Gierasch, P. J., Flasar, F. M., Nixon, C. A., 2008a. Observation of a tilt of Titan's middle-atmospheric superrotation. Icarus 197, 549-555.

Achterberg, R. K., Conrath, B. J., Gierasch, P. J., Flasar, F. M., Nixon, C. A., 2008b. Titan's middle-atmospheric temperatures and dynamics observed by 
the Cassini Composite Infrared Spectrometer. Icarus 194, 263-277.

Achtermann, H. J., Magnus, G., Bose, T. K., 1991. Refractivity virial coefficients of gaseous $\mathrm{CH}_{4}, \mathrm{C}_{2} \mathrm{H}_{4}, \mathrm{C}_{2} \mathrm{H}_{6}, \mathrm{CO}_{2}, \mathrm{SF}_{6}, \mathrm{H}_{2}, \mathrm{~N}_{2}, \mathrm{He}$, and $\mathrm{Ar}$. J. Chem. Phys. 94, 5669-5684.

Baum, W. A., Code, A. D., 1953. A photometric observation of the occultation of $\sigma$ Arietis by Jupiter. Astronomical Journal 58, 108-112.

Bender, E., 1971. Die Berechnung der Verdampfungsgleichgewichte von Mehrstoffsystemen bei hohen Drcken. Chemie. Ing. Techn. 44, 576-582.

Bender, E., 1973. An equation of state for predicting vapour-liquid equilibria of the system $\mathrm{N}_{2}-\mathrm{Ar}-\mathrm{O}_{2}$. Cryogenics 13, 11-18.

Borysow, A., Frommhold, L., 1986. Collision-induced rototranslational absorption spectra of $N_{2}-N_{2}$ pairs for temperatures from 50 to $300 \mathrm{~K}$. Astrophys. J. 311, 1043-1057.

Bühner, K., Maurer, G., Bender, E., 1981. Pressure-enthalpy diagrams for methane, ethane, propane, ethylene, and propylene. Cryogenics 21, 157164.

Cahoy, K. L., Hinson, D. P., Tyler, G. L., 2006. Radio science measurements of atmospheric refractivity with Mars Global Surveyor. Journal of Geophysical Research (Planets) 111, 5003-5014.

Coustenis, A., Ferri, F., Colombatti, G., Fulchignoni, M., Barucci, A., Conrath, B. J., Flasar, F. M., Achterberg, R., Nixon, C., Zarnecki, J., HASI Investigation Team, CIRS Investigation Team, 2005. Titan's atmospheric temperature profile from Cassini - Huygens ASI and CIRS measurements. Geophys. Res. Abstracts 7, 05756.

Eshleman, V. R., 1975. Jupiter's atmosphere - Problems and potential of radio occultation. Science 189, 876-878.

Essen, L., Froome, K. D., 1951. The Refractive Indices and Dielectric Con- 
stants of Air and its Principal Constituents at 24,000 Mc/s. Proc. Phys. Soc. London Ser. B 64, 862-875.

Ferri, F., Coustenis, A., Marten, A., Colombatti, G., Fulchignoni, M., Flasar, F., the HASI and CIRS teams, 2006. Titan's atmospheric temperature profile from Cassini - Huygens ASI and CIRS measurements. Geophys. Res. Abstracts 8, 09064.

Fjeldbo, G., Eshleman, V. R., 1969. Atmosphere of Venus as Studied with the Mariner 5 Dual Radio-Frequency Occultation Experiment. Radio Science 4, 879-897.

Fjeldbo, G., Kliore, A. J., Eshleman, V. R., 1971. The neutral atmosphere of Venus as studied with the Mariner $\mathrm{V}$ radio occultation experiments. Astronomical Journal 76, 123-140.

Flasar, F. M., Achterberg, R. K., 2009. The structure and dynamics of Titan's middle atmosphere. Phil. Trans. Roy. Soc. London A 367, 649-664.

Flasar, F. M., Achterberg, R. K., Conrath, B. J., Gierasch, P. J., Kunde, V. G., Nixon, C. A., Bjoraker, G. L., Jennings, D. E., Romani, P. N., Simon-Miller, A. A., Bézard, B., Coustenis, A., Irwin, P. G. J., Teanby, N. A., Brasunas, J., Pearl, J. C., Segura, M. E., Carlson, R. C., Mamoutkine, A., Schinder, P. J., Barucci, A., Courtin, R., Fouchet, T., Gautier, D., Lellouch, E., Marten, A., Prangé, R., Vinatier, S., Strobel, D. F., Calcutt, S. B., Read, P. L., Taylor, F. W., Bowles, N., Samuelson, R. E., Orton, G. S., Spilker, L. J., Owen, T. C., Spencer, J. R., Showalter, M. R., Ferrari, C., Abbas, M. M., Raulin, F., Edgington, S., Ade, P., Wishnow, E. H., 2005. Titan's Atmospheric Temperatures, Winds, and Composition. Science 308, 975-978.

Flasar, F. M., Kunde, V. G., Abbas, M. M., Achterberg, R. K., Ade, P., Barucci, A., B'ezard, B., Bjoraker, G. L., Brasunas, J. C., Calcutt, S., Carlson, R., C'esarsky, C. J., Conrath, B. J., Coradini, A., Courtin, R., 
Coustenis, A., Edberg, S., Edgington, S., Ferrari, C., Fouchet, T., Gautier, D., Gierasch, P. J., Grossman, K., Irwin, P., Jennings, D. E., Lellouch, E., Mamoutkine, A. A., Marten, A., Meyer, J. P., Nixon, C. A., Orton, G. S., Owen, T. C., Pearl, J. C., Prang'e, R., Raulin, F., Read, P. L., Romani, P. N., Samuelson, R. E., Segura, M. E., Showalter, M. R., Simon-Miller, A. A., Smith, M. D., Spencer, J. R., Spilker, L. J., Taylor, F. W., 2004. Exploring The Saturn System In The Thermal Infrared: The Composite Infrared Spectrometer. Space Science Reviews 115, 169-297.

Flasar, F. M., Samuelson, R. E., Conrath, B. J., 1981. Titan's atmosphere Temperature and dynamics. Nature 292, 693-698.

French, R. G., Gierasch, P. J., 1976. Diffraction calculation of occultation light curves in the presence of an isothermal atmosphere. Astronomical Journal 81, 445-451.

French, R. G., Lovelace, R. V. F., 1983. Strong turbulence and atmospheric waves in stellar occultations. Icarus 56, 122-146.

Fulchignoni, M., Ferri, F., Angrilli, F., Ball, A. J., Bar-Nun, A., Barucci, M. A., Bettanini, C., Bianchini, G., Borucki, W., Colombatti, G., Coradini, M., Coustenis, A., Debei, S., Falkner, P., Fanti, G., Flamini, E., Gaborit, V., Grard, R., Hamelin, M., Harri, A. M., Hathi, B., Jernej, I., Leese, M. R., Lehto, A., Lion Stoppato, P. F., López-Moreno, J. J., Mäkinen, T., McDonnell, J. A. M., McKay, C. P., Molina-Cuberos, G., Neubauer, F. M., Pirronello, V., Rodrigo, R., Saggin, B., Schwingenschuh, K., Seiff, A., Simões, F., Svedhem, H., Tokano, T., Towner, M. C., Trautner, R., Withers, P., Zarnecki, J. C., 2005. In situ measurements of the physical characteristics of Titan's environment. Nature 438, 785-791.

Goody, R. M., Yung, Y. L., 1989. Atmospheric radiation : theoretical basis. Oxford University Press. 
Haugstad, B. S., 1978. Effects of the inhomogeneous background on radiation propagating through turbulent planetary atmospheres. Radio Science 13, $435-440$.

Hinson, D. P., Flasar, F. M., J., K. A., Schinder, P. J., Twicken, J. D., G., H. R., 1997. Jupiter's ionosphere: Results from the first Galileo radio occultation experiment. Geophysical Research Letters 24 (17), 2107-2110.

Hinson, D. P., Jenkins, J. M., 1995. Magellan radio occultation measurements of atmospheric waves on Venus. Icarus 114, 310-327.

Ho, W., Kaufman, I. A., Thaddeus, P., 1968. Pressure Induced Microwave Absorption in $N_{2}$. Journal of Chemical Physics 49, 3627-3631.

Hubbard, W. B., Sicardy, B., Miles, R., Hollis, A. J., Forrest, R. W., Nicolson, I. K. M., Appleby, G., Beisker, W., Bittner, C., Bode, H.-J., Bruns, M., Denzau, H., Nezel, M., Riedel, E., Struckmann, H., Arlot, J. E., Roques, F., Sevre, F., Thuillot, W., Hoffmann, M., Geyer, E. H., Buil, C., Colas, F., Lecacheux, J., Klotz, A., Thouvenot, E., Vidal, J. L., Carreira, E., Rossi, F., Blanco, C., Cristaldi, S., Nevo, Y., Reitsema, H. J., Brosch, N., Cernis, K., Zdanavicius, K., Wasserman, L. H., Hunten, D. M., Gautier, D., Lellouch, E., Yelle, R. V., Rizk, B., Flasar, F. M., Porco, C. C., Toublanc, D., Corugedo, G., 1993. The occultation of 28 SGR by Titan. Astronomy and Astrophysics 269, 541-563.

Jenkins, J. M., Steffes, P. G., Hinson, D. P., Twicken, J. D., Tyler, G. L., 1994. Radio occultation studies of the Venus atmosphere with the Magellan spacecraft. 2: Results from the October 1991 experiments. Icarus 110, 79-94.

Karayel, E. T., Hinson, D. P., 1997. Sub-Fresnel-scale vertical resolution in atmospheric profiles from radio occultation. Radio Science 32, 411-423.

Kliore, A. J., Anderson, J. D., Armstrong, J. W., Asmar, S. W., Hamilton, C. L., Rappaport, N. J., Wahlquist, H. D., Ambrosini, R., Flasar, F. M., 
French, R. G., Iess, L., Marouf, E. A., Nagy, A. F., 2004. Cassini Radio Science. Space Science Reviews 115, 1-4.

Lindal, G. F., 1992. The atmosphere of Neptune - an analysis of radio occultation data acquired with Voyager 2. Astronomical Journal 103, 967-982. Lindal, G. F., Lyons, J. R., Sweetnam, D. N., Eshleman, V. R., Hinson, D. P., 1987. The atmosphere of Uranus - Results of radio occultation measurements with Voyager 2. Journal of Geophysical Research 92, 14987-15001.

Lindal, G. F., Sweetnam, D. N., Eshleman, V. R., 1985. The atmosphere of Saturn - an analysis of the Voyager radio occultation measurements. Astron. J. 90, 1136-1146.

Lindal, G. F., Wood, G. E., Hotz, H. B., Sweetnam, D. N., Eshleman, V. R., Tyler, G. L., 1983. The atmosphere of Titan - an analysis of the Voyager 1 radio occultation measurements. Icarus 53, 348-363.

Lipa, B., Tyler, G. L., 1979. Statistical and computational uncertainties in atmospheric profiles from radio occultation - Mariner 10 at Venus. Icarus 39, 192-208.

Marouf, E. A., Tyler, G. L., Rosen, P. A., 1986. Profiling Saturn's rings by radio occultation. Icarus $68,128-166$.

Niemann, H. B., Atreya, S. K., Bauer, S. J., Carignan, G. R., Demick, J. E., Frost, R. L., Gautier, D., Haberman, J. A., Harpold, D. N., Hunten, D. M., Israel, G., Lunine, J. I., Kasprzak, W. T., Owen, T. C., Paulkovich, M., Raulin, F., Raaen, E., Way, S. H., 2005. The abundances of constituents of Titan's atmosphere from the GCMS instrument on the Huygens probe. Nature 438, 779-784.

Niemann, H. B., Atreya, S. K., Demick, J. E., Gautier, D., Haberman, J. A., Harpold, D. N., Kasprzak, W. T., Lunine, J. I., Owen, T. C., Raulin, F., 2010. Composition of Titan's lower atmosphere and simple surface volatiles 
as measured by the Cassini-Huygens probe gas chromatograph mass spectrometer experiment. J. Geophys. Res. 115, E12006.

Omar, M. H., Dokoupil, Z., Schroten, H. G. M., 1962. Determination of the solid-liquid equilibrium diagram for the nitrogen-methane system. Physica $28,309-329$.

Orcutt, R. H., Cole, R. H., 1967. Dielectric Constants of Imperfect Gases. III. Atomic gases, Hydrogen, and Nitrogen. J. Chem. Phys 46, 697-702.

Phinney, R. A., Anderson, D. L., 1968. On the radio occulataion method for studying planetary atmospheres. J. Geophys. Res. 73, 1819-1927.

Samuelson, R. E., 1983. Radiative equilibrium model of Titan's atmosphere. Icarus 53, 364-387.

Schunk, R. W., Nagy, A. F., 2000. Ionospheres: Physics, Plasma Physics, and Chemistry. Cambridge University Press, Cambridge (UK) and New York.

Shemansky, D. E., Stewart, A. I. F., West, R. A., Esposito, L. W., Hallett, J. T., Liu, X., 2005. The Cassini UVIS Stellar Probe of the Titan Atmosphere. Science 308, 978-982.

Sicardy, B., Colas, F., Widemann, T., Bellucci, A., Beisker, W., Kretlow, M., Ferri, F., Lacour, S., Lecacheux, J., Lellouch, E., Pau, S., Renner, S., Roques, F., Fienga, A., Etienne, C., Martinez, C., Glass, I. S., Baba, D., Nagayama, T., Nagata, T., Itting-Enke, S., Bath, K.-L., Bode, H.-J., Bode, F., Lüdemann, H., Lüdemann, J., Neubauer, D., Tegtmeier, A., Tegtmeier, C., Thomé, B., Hund, F., deWitt, C., Fraser, B., Jansen, A., Jones, T., Schoenau, P., Turk, C., Meintjies, P., Hernandez, M., Fiel, D., Frappa, E., Peyrot, A., Teng, J. P., Vignand, M., Hesler, G., Payet, T., Howell, R. R., Kidger, M., Ortiz, J. L., Naranjo, O., Rosenzweig, P., Rapaport, M., 2006. The two Titan stellar occultations of 14 November 2003. Journal of Geophysical Research (Planets) 111, 11-37. 
Tokano, T., Ferri, F., Colombatti, G., Mäkinen, T., Fulchignoni, M., 2006. Titan's planetary boundary layer structure at the Huygens landing site. Journal of Geophysical Research (Planets) 111, 8007-8017. 
Table 1: Titan Radio Occultations through 2006

\begin{tabular}{|l|l|l|l|l|}
\hline \multirow{2}{*}{} & \multicolumn{2}{|c|}{ Ingress } & \multicolumn{2}{c|}{ Egress } \\
\cline { 2 - 6 } & Latitude & $\begin{array}{l}\text { Distance to } \\
\text { Limb }(\mathrm{km})^{1}\end{array}$ & Latitude & $\begin{array}{l}\text { Distance to } \\
\text { Limb }(\mathrm{km})^{1}\end{array}$ \\
\hline Voyager 1 & $6.2^{\circ} \mathrm{N}$ & 8700 & $8.5^{\circ} \mathrm{S}$ & 19500 \\
\hline 12 November $1979^{2}$ & \multicolumn{5}{|l}{} \\
\hline Cassini & $31.4^{\circ} \mathrm{S}$ & 3745 & $52.8^{\circ} \mathrm{S}$ & 5549 \\
\hline T12: 19 March 2006 & $32.7^{\circ} \mathrm{S}$ & 4027 & $34.3^{\circ} \mathrm{S}$ & 4183 \\
\hline T14: 20 May 2006 & & & & \\
\hline
\end{tabular}

$\overline{1}$ Spacecraft distance to the limb corresponds to the time when the ray from the spacecraft to the receiving station on the Earth just grazes the solid surface of Titan, i.e., the "last" ray on ingress or the "first" ray on egress. Latitude is that corresponding to the periapsis of of the grazing ray. Dates correspond to Universal Time on reception.

2 Voyager 1 results from Lindal et al. (1983) 


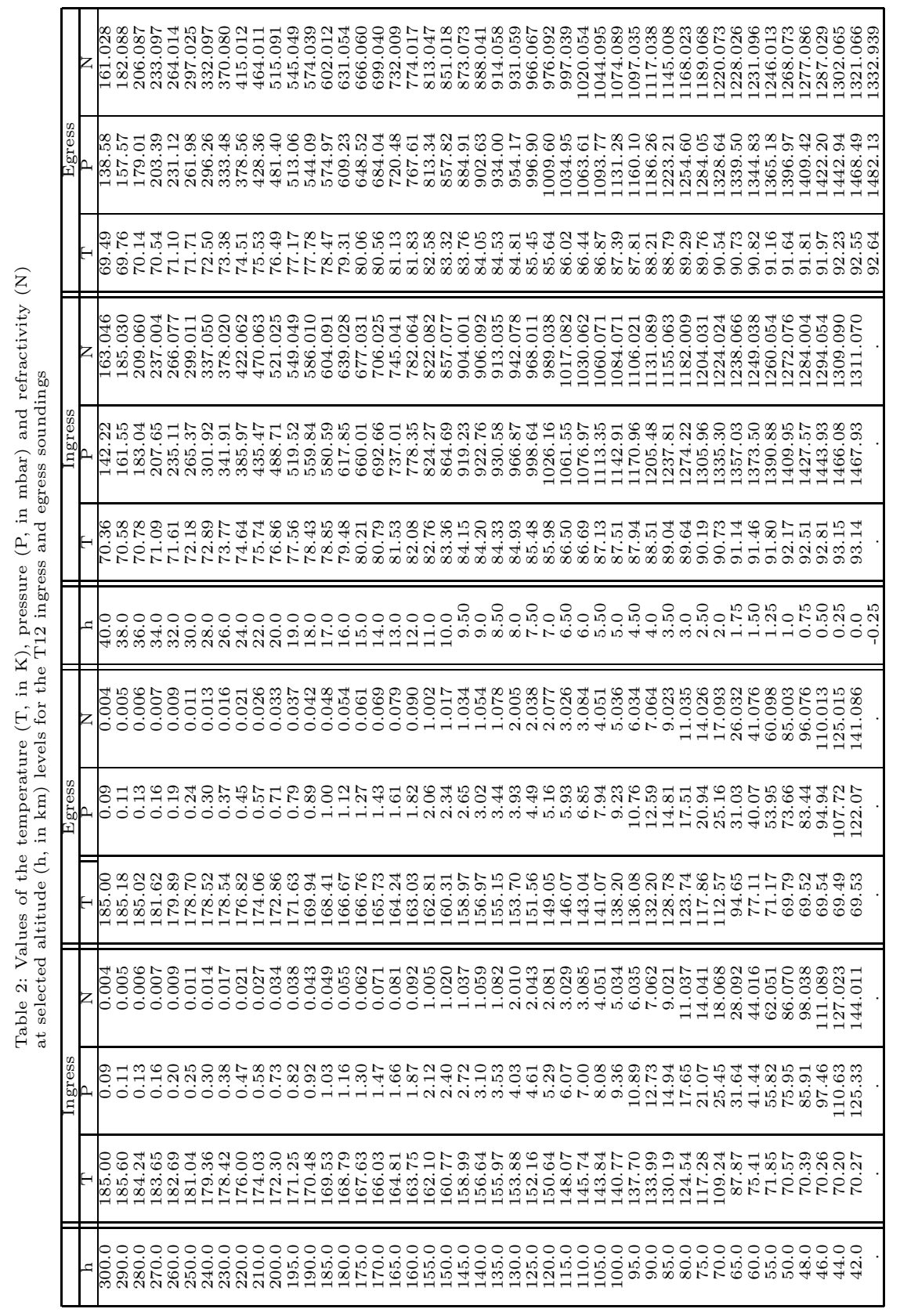




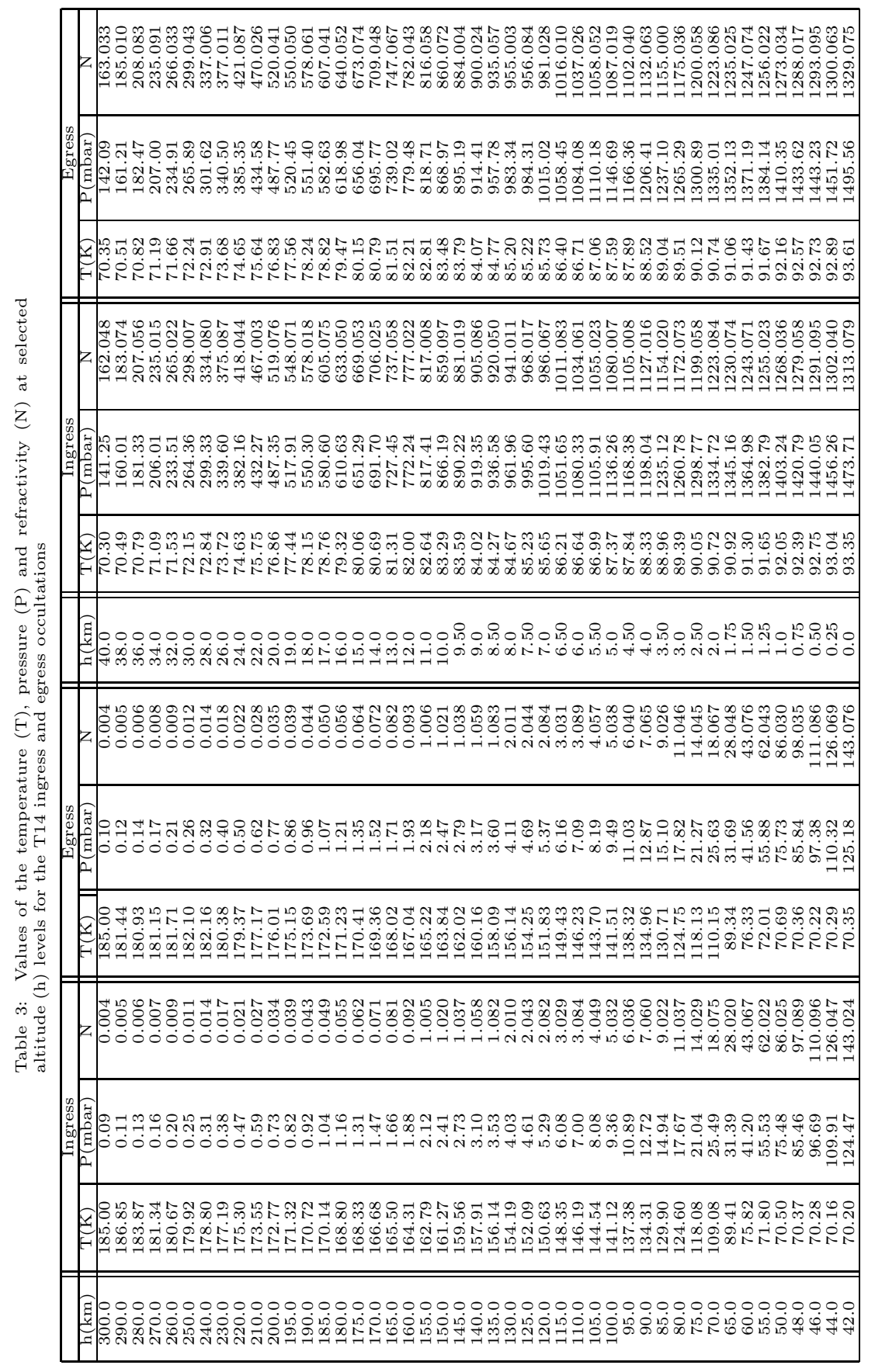




\section{List of Figures}

1 The power in the signal for the T12 ingress (a), T12 egress(b), T14 ingress (c), and T14 egress (d) soundings in the S, X, and Ka bands. The T12 power shown here was recorded at DSS-63 (S, X) and DSS-55 (Ka), and the T14 power was recorded at the same antennas. The black lines are a prediction of the power loss due to refractive defocusing computed as the refractivity profile was obtained. The $\mathrm{S}$ band power loss is essentially all due to refractive defocusing. Both X and Ka are visibly affected by $N_{2}$ absorption, with the Ka band power lost altogether within $\sim 10 \mathrm{~km}$ of the surface. The time axis is Earth received time in seconds past UT midnight on March 19, 2006 for T12 and seconds past UT midnight on May 20, 2006 for T14.

2 Latitude vs. longitude for each of the soundings discussed in this paper: (a) T12 ingress, (b) T12 egress (c) T14 ingress, (d) T14 egress. The (north) latitude and (west) longitude of the point on each ray closest to the surface of Titan (the ray periapsis) is shown as subsequent rays approach the surface of Titan (or in the case of egress soundings, recede from the surface). The circles denote the location of the indicated altitudes in $\mathrm{km}$ relative to a sphere of radius $2575 \mathrm{~km}$.

3 Top. Temperature-altitude profiles for the T12 occultation, assuming a uniformly mixed atmosphere with mole fractions $0.985 \mathrm{~N}_{2}$ and $0.015 \mathrm{CH}_{4}$. The temperature was set to $185 \mathrm{~K}$ at $300 \mathrm{~km}$. Random errors $(3 \sigma)$ from thermal noise and spacecraft velocity errors are depicted on the right. The ephemeris was adjusted by using an along track shift so that the stratospheric profile agreed with profiles from CIRS (Achterberg et al., $2008 \mathrm{~b}$ ), in an attempt to minimize the effects of the velocity errors. The ingress ephemeris was shifted by $-0.005 \mathrm{~s}$, and the egress $-0.014 \mathrm{~s}$. Both of these shifts produce velocity shifts outside the $1 \sigma$ quoted error for the ephemeris, although they are well within the $3 \sigma$ level. Bottom. The same, except for T14. The shift for the ingress was $-0.005 \mathrm{~s}$, while that for the egress was -0.007 . Both of these shift produce velocity shifts of $\sim 1 \mathrm{~mm} / \mathrm{s}$, well within the quoted errors for the ephemeris. 
4 Top: The effect of starting the hydrostatic equilibrium computation at three different temperatures at $300 \mathrm{~km}$. Note that the difference is small below $\sim 150 \mathrm{~km}$ but noticeable above that. Bottom: We start the integration at $148 \mathrm{~km}$ at $172.5 \mathrm{~K}$, approximately the same as the top of the HASI direct measurements. We compare our profile to the HASI direct measurements below $148 \mathrm{~km}$. Both panels show the T14 egress occultation using $16 \mathrm{kHz} \mathrm{X}$ band data.

5 Doppler residual frequencies for DSS-63, X band, $16 \mathrm{kHz}$ data, for the T14 ingress occultation.

6 The ephemeris was shifted by the indicated times in seconds to produce these temperature profiles. This example is the T14 egress occultation using $16 \mathrm{kHz} \mathrm{X}$ band data.

$7 \quad$ The results of a Monte Carlo study using the covariance matrix for the T14 egress occultation, using $16 \mathrm{kHz} \mathrm{X}$ band data. The velocity was shifted in a random direction by 3 times the covariance matrix elements. 100 cases were done. The solid line is the T14 egress profile, and the dotted lines encompass the $3 \sigma$ error envelope. The maximum error is $\pm 20 \mathrm{~K}$.

8 Temperatures from the T12 and T14 occultations, obtained by adjusting the the spacecraft timing on ingress and egress until the retrieved temperature profiles in the upper stratosphere matched the CIRS zonal averages (see Fig. 3 caption). Note that the vertical axis in this case is pressure, not altitude.

9 The effects that random noise of magnitude similar to that already existing in the signal has on the temperature - altitude profiles, for T14 egress, $16 \mathrm{kHz} \mathrm{X}$ band data, DSS-63. The solid line is the T14 egress profile, and the dotted lines encompass the $3 \sigma$ error envelope. 
10 (a) Retrieved profiles of temperature from the T12 and T14 soundings for an assumed uniformly mixed atmosphere with mole fractions of $\mathrm{N}_{2}: 0.986$ and $\mathrm{CH}_{4}$ : 0.015. (b). T14 profiles compared to those retrieved from Voyager radio occultations (rescaled to an atmosphere with the same mole fraction of $\mathrm{CH}_{4}$ ) and temperatures from the HASI experiment. The 1- $\sigma$ errors indicated are from thermal noise only. (c), (d) Enlargement of (a), (b) in the troposphere and tropopause region. (e), (f) Enlargement of (c), (d) in the lowest $10 \mathrm{~km}$ of the troposphere. The retrieved T14 ingress temperature assuming the variable profile of gaseous $\mathrm{CH}_{4}$ obtained by the Huygens GCMS experiment is also shown in (f). The latitudes indicated for each occultation correspond to the periapsis of the rays grazing Titan's surface. Dry adiabats are shown.

11 Vertical gradients of temperature in the lowest $10 \mathrm{~km}$ for the T12 and T14 soundings. The vertical straight line in each panel corresponds to the dry adiabat.

12 Retrieved temperatures and their vertical gradients from Abel inversions for an isothermal atmosphere with a sinusoidal perturbation with fractional amplitude indicated in each panel. Temperatures and gradients are normalized by the mean isothermal temperature, $T_{0}$. Dotted curves. Input perturbation temperatures, used in the forward ray-tracing calculation of light curves. Solid curves. Temperatures retrieved from the light curves using Abel inversions and the associated vertical gradients. Zero altitude corresponds to the half-power point on the light curve. The model calculations do not include a lower bounding surface. After French and Lovelace (1983). 


\section{Figures}

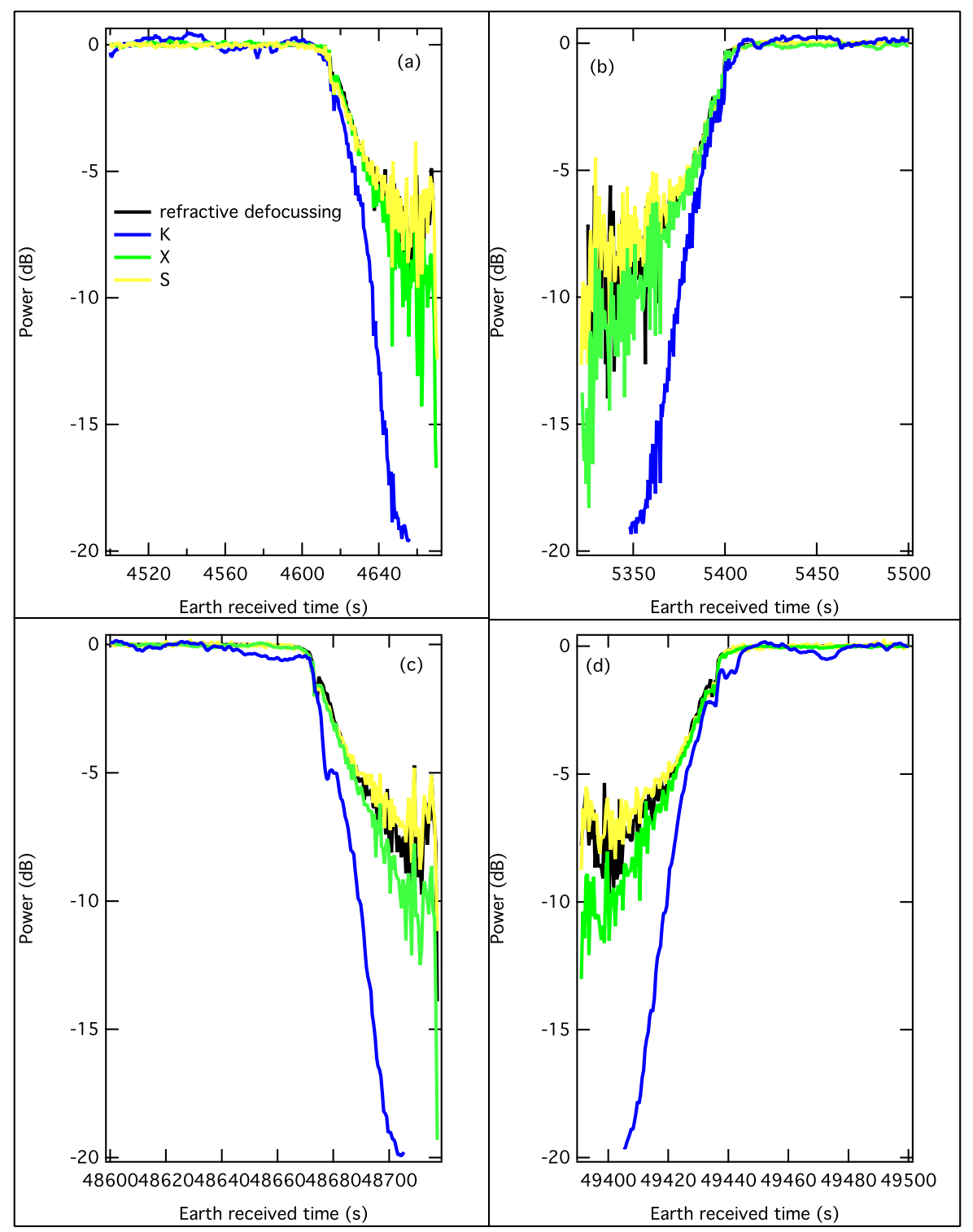

Figure 1. The power in the signal for the T12 ingress (a), T12 egress(b), T14 ingress (c), and T14 egress (d) soundings in the S, X, and Ka bands. The T12 power shown here was recorded at DSS-63 $(\mathrm{S}, \mathrm{X})$ and DSS-55 (Ka), and the T14 power was recorded at the same antennas. The black lines are a prediction of the power loss due to refractive defocusing computed as the refractivity profile was obtained. The $\mathrm{S}$ band power loss is essentially all due to refractive defocusing. Both $\mathrm{X}$ and Ka are visibly affected by $N_{2}$ absorption, with the Ka band power lost altogether within $\sim 10 \mathrm{~km}$ of the surface. The time axis is Earth received time in seconds past UT midnight on March 19, 2006 for T12 and seconds past UT midnight on May 20, 2006 for T14. 

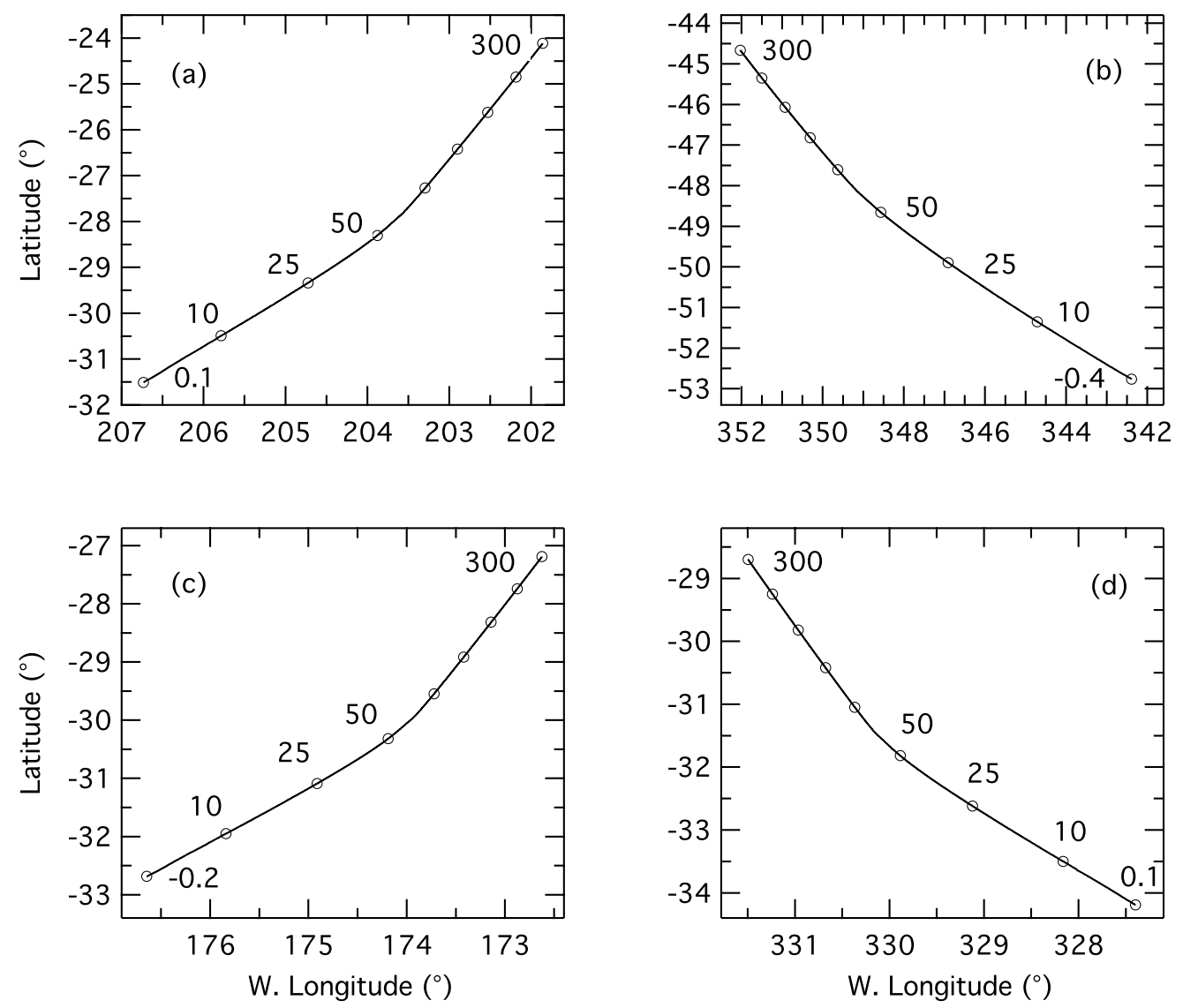

Figure 2. Latitude vs. longitude for each of the soundings discussed in this paper: (a) T12 ingress, (b) T12 egress (c) T14 ingress, (d) T14 egress. The (north) latitude and (west) longitude of the point on each ray closest to the surface of Titan (the ray periapsis) is shown as subsequent rays approach the surface of Titan (or in the case of egress soundings, recede from the surface). The circles denote the location of the indicated altitudes in $\mathrm{km}$ relative to a sphere of radius $2575 \mathrm{~km}$. 

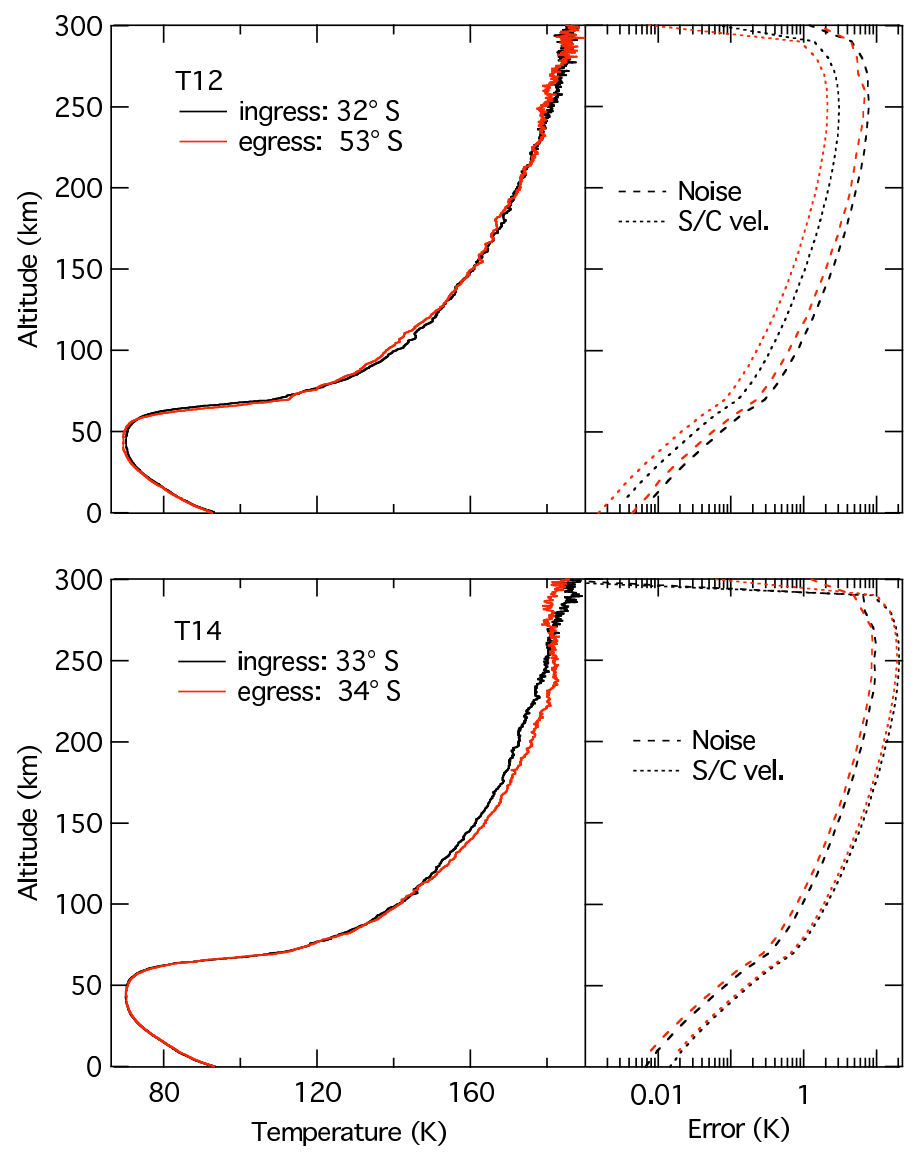

Figure 3. Top. Temperature-altitude profiles for the T12 occultation, assuming a uniformly mixed atmosphere with mole fractions $0.985 \mathrm{~N}_{2}$ and $0.015 \mathrm{CH}_{4}$. The temperature was set to $185 \mathrm{~K}$ at $300 \mathrm{~km}$. Random errors $(3 \sigma)$ from thermal noise and spacecraft velocity errors are depicted on the right. The ephemeris was adjusted by using an along track shift so that the stratospheric profile agreed with profiles from CIRS (Achterberg et al., 2008b), in an attempt to minimize the effects of the velocity errors. The ingress ephemeris was shifted by $-0.005 \mathrm{~s}$, and the egress -0.014 s. Both of these shifts produce velocity shifts outside the $1 \sigma$ quoted error for the ephemeris, although they are well within the $3 \sigma$ level. Bottom. The same, except for T14. The shift for the ingress was $-0.005 \mathrm{~s}$, while that for the egress was -0.007 . Both of these shift produce velocity shifts of $\sim 1 \mathrm{~mm} / \mathrm{s}$, well within the quoted errors for the ephemeris. 

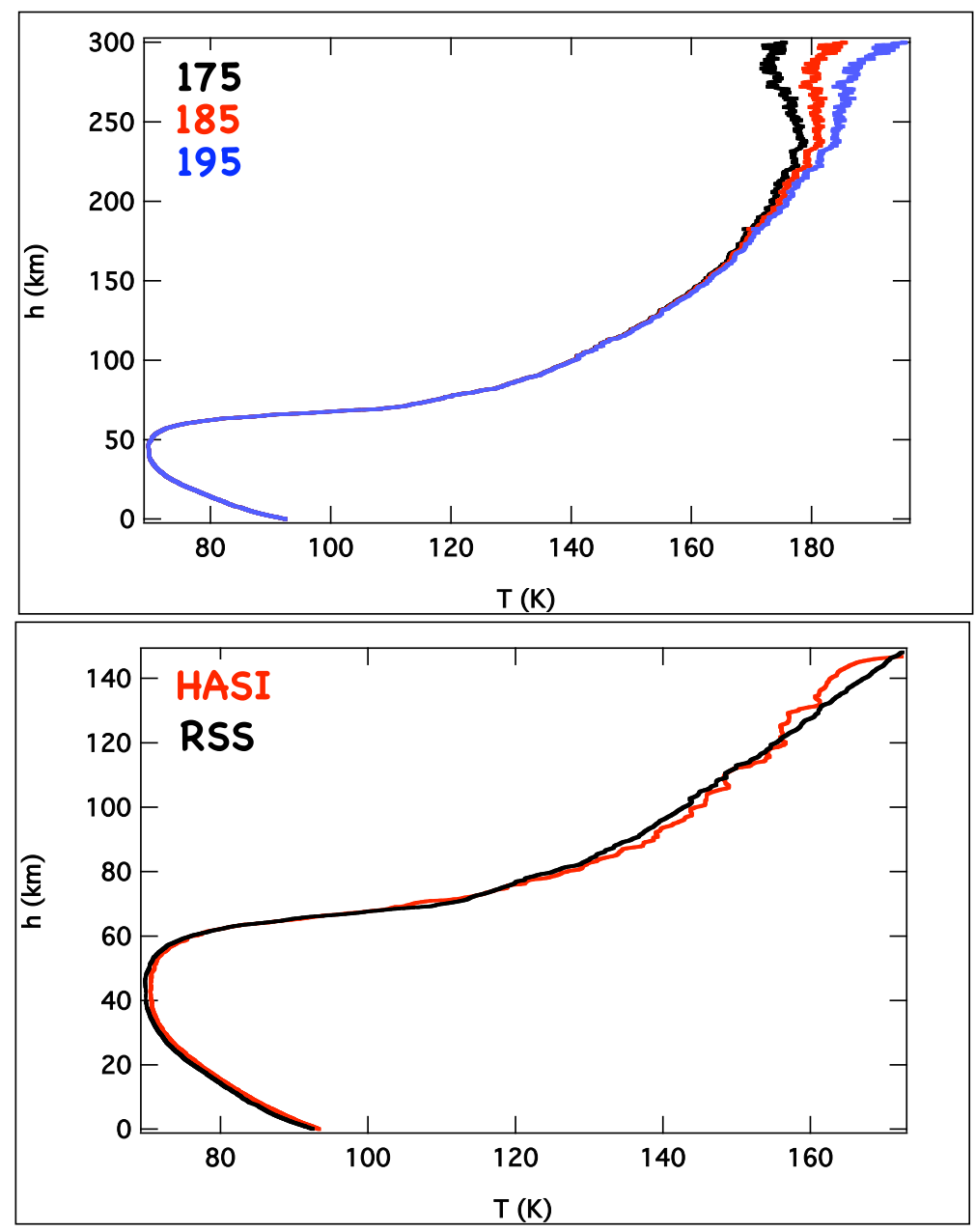

Figure 4. Top: The effect of starting the hydrostatic equilibrium computation at three different temperatures at $300 \mathrm{~km}$. Note that the difference is small below $\sim 150 \mathrm{~km}$ but noticeable above that. Bottom: We start the integration at $148 \mathrm{~km}$ at $172.5 \mathrm{~K}$, approximately the same as the top of the HASI direct measurements. We compare our profile to the HASI direct measurements below $148 \mathrm{~km}$. Both panels show the T14 egress occultation using $16 \mathrm{kHz} \mathrm{X}$ band data. 


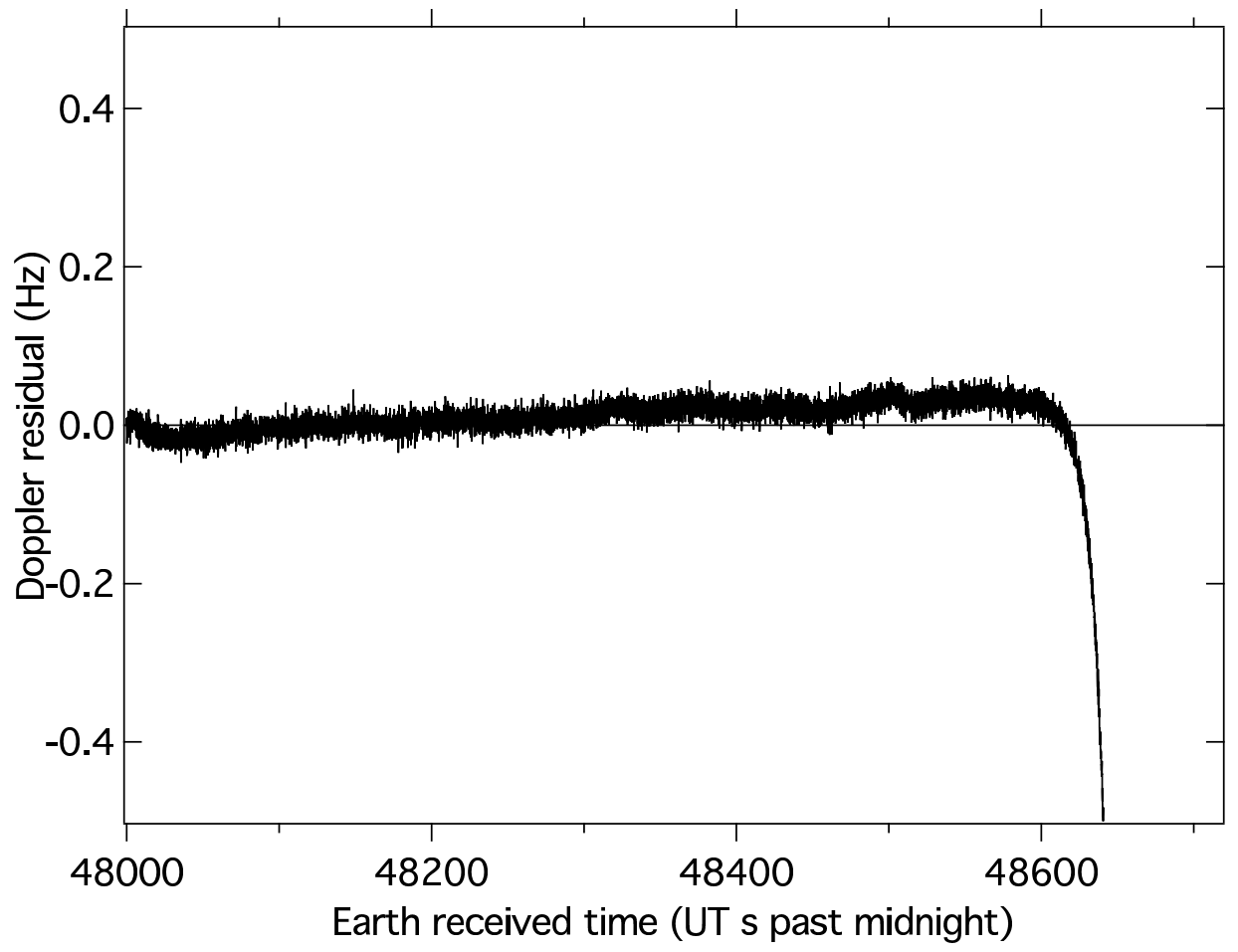

Figure 5. Doppler residual frequencies for DSS-63, X band, $16 \mathrm{kHz}$ data, for the T14 ingress occultation. 


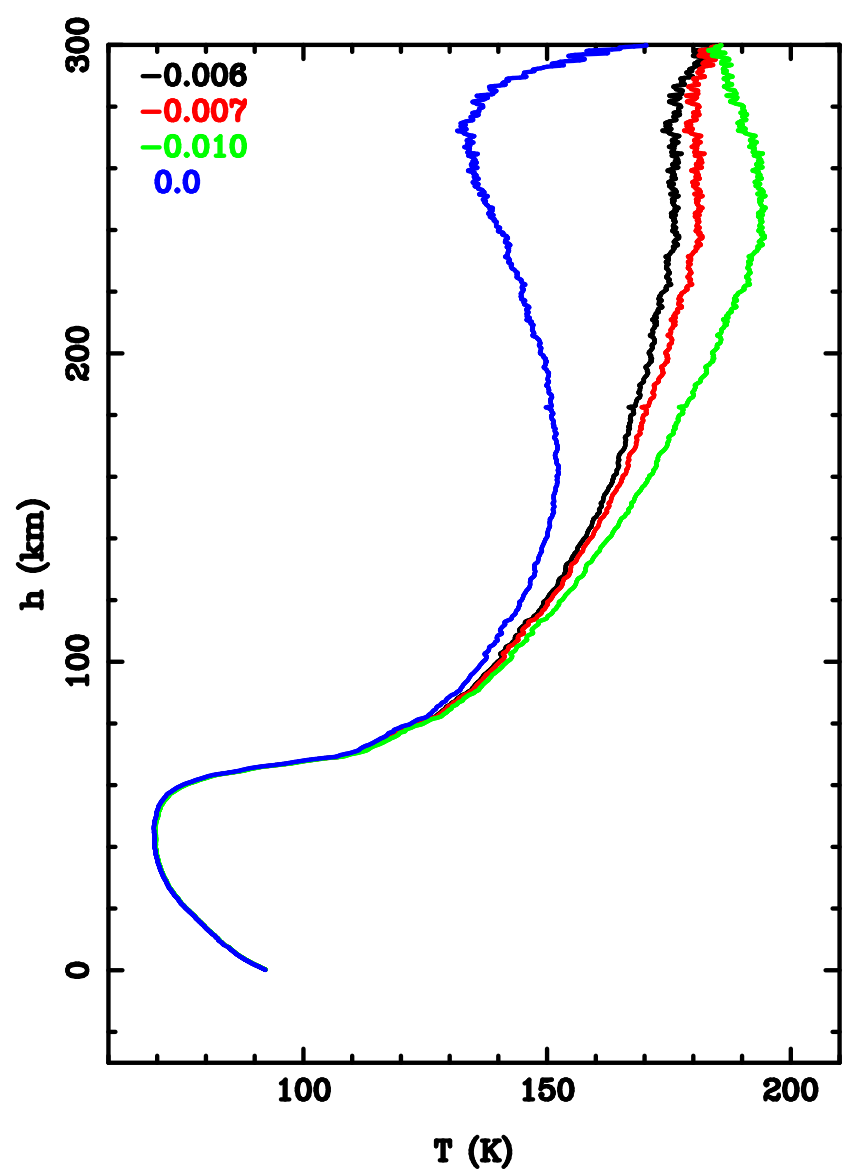

Figure 6 . The ephemeris was shifted by the indicated times in seconds to produce these temperature profiles. This example is the T14 egress occultation using $16 \mathrm{kHz}$ $\mathrm{X}$ band data. 


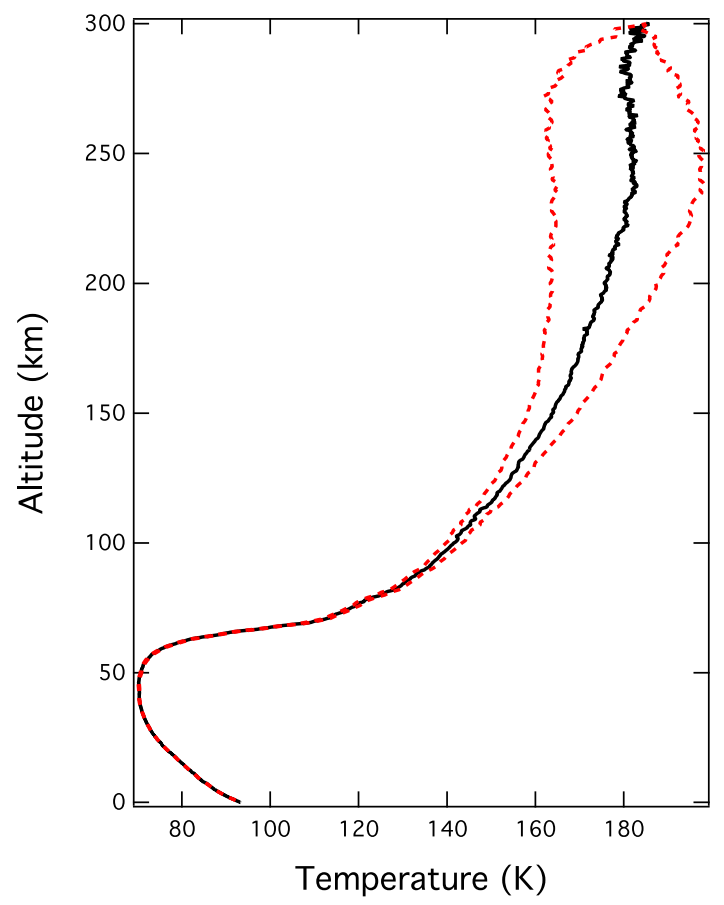

Figure 7. The results of a Monte Carlo study using the covariance matrix for the T14 egress occultation, using $16 \mathrm{kHz} \mathrm{X}$ band data. The velocity was shifted in a random direction by 3 times the covariance matrix elements. 100 cases were done. The solid line is the T14 egress profile, and the dotted lines encompass the $3 \sigma$ error envelope. The maximum error is $\pm 20 \mathrm{~K}$. 

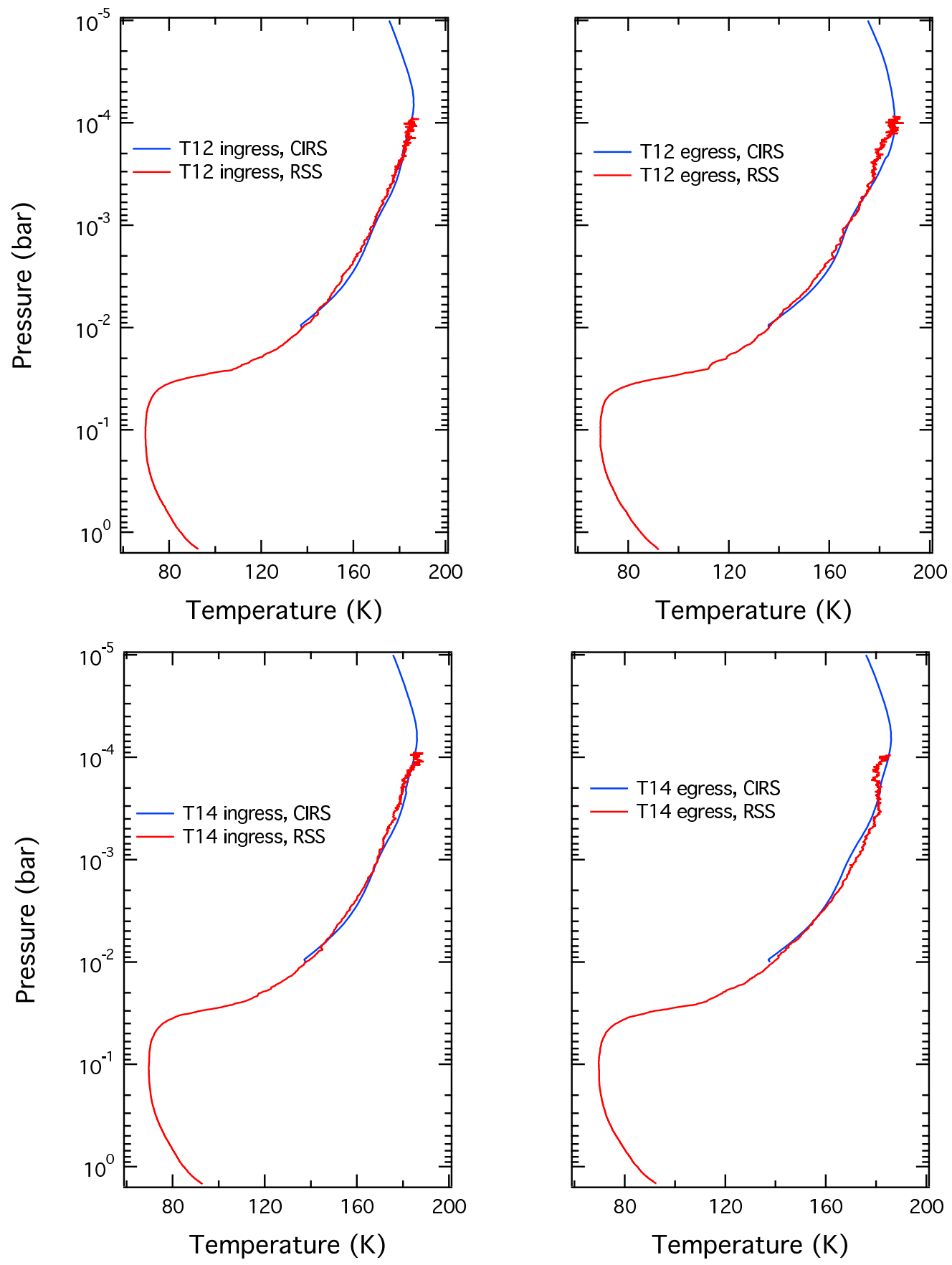

Figure 8. Temperatures from the T12 and T14 occultations, obtained by adjusting the the spacecraft timing on ingress and egress until the retrieved temperature profiles in the upper stratosphere matched the CIRS zonal averages (see Fig. 3 caption). Note that the vertical axis in this case is pressure, not altitude. 


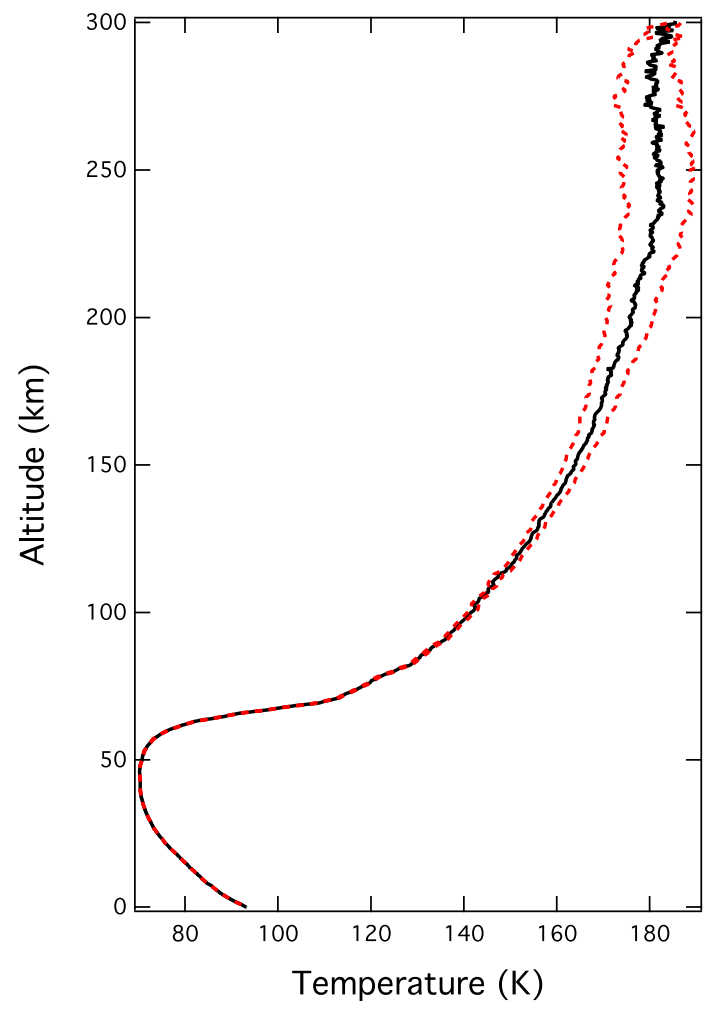

Figure 9. The effects that random noise of magnitude similar to that already existing in the signal has on the temperature - altitude profiles, for T14 egress, $16 \mathrm{kHz}$ X band data, DSS-63. The solid line is the T14 egress profile, and the dotted lines encompass the $3 \sigma$ error envelope. 


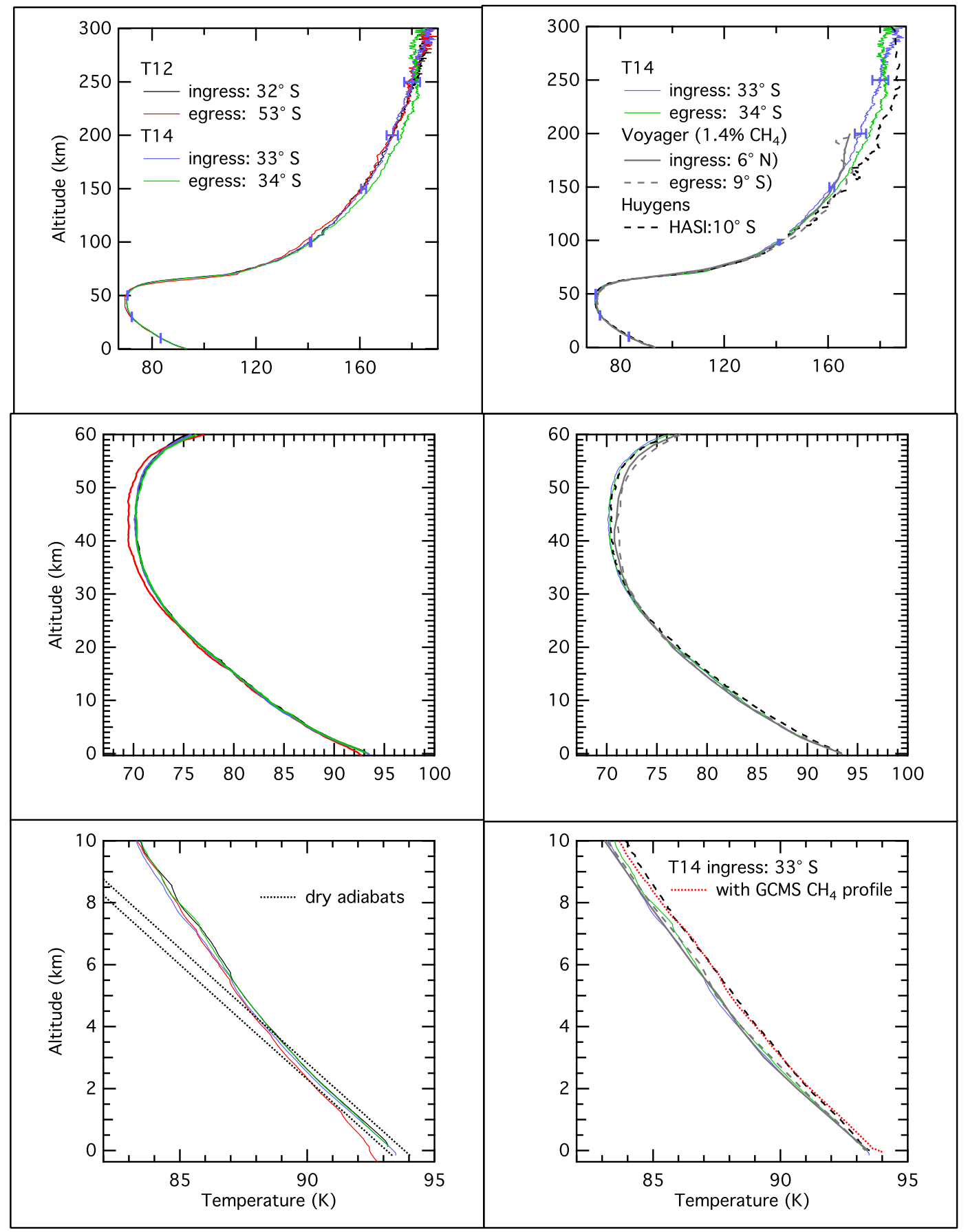

Figure 10. (a) Retrieved profiles of temperature from the T12 and T14 soundings for an assumed uniformly mixed atmosphere with mole fractions of $\mathrm{N}_{2}: 0.986$ and $\mathrm{CH}_{4}$ : 0.015. (b). T14 profiles compared to those retrieved from Voyager radio occultations (rescaled to an atmosphere with the same mole fraction of $\mathrm{CH}_{4}$ ) and temperatures from the HASI experiment. The 1- $\sigma$ errors indicated are from thermal noise only. (c), (d) Enlargement of (a), (b) in the troposphere and tropopause region. (e), (f) Enlargement of (c), (d) in the lowest $10 \mathrm{~km}$ of the troposphere. The retrieved T14 ingress temperature assuming the variable profile of gaseous $\mathrm{CH}_{4}$ obtained by the Huygens GCMS experiment is also shown in (f). The latitudes indicated for each occultation correspond to the periapsis of the rays grazing Titan's surface. Dry adiabats are shown. 


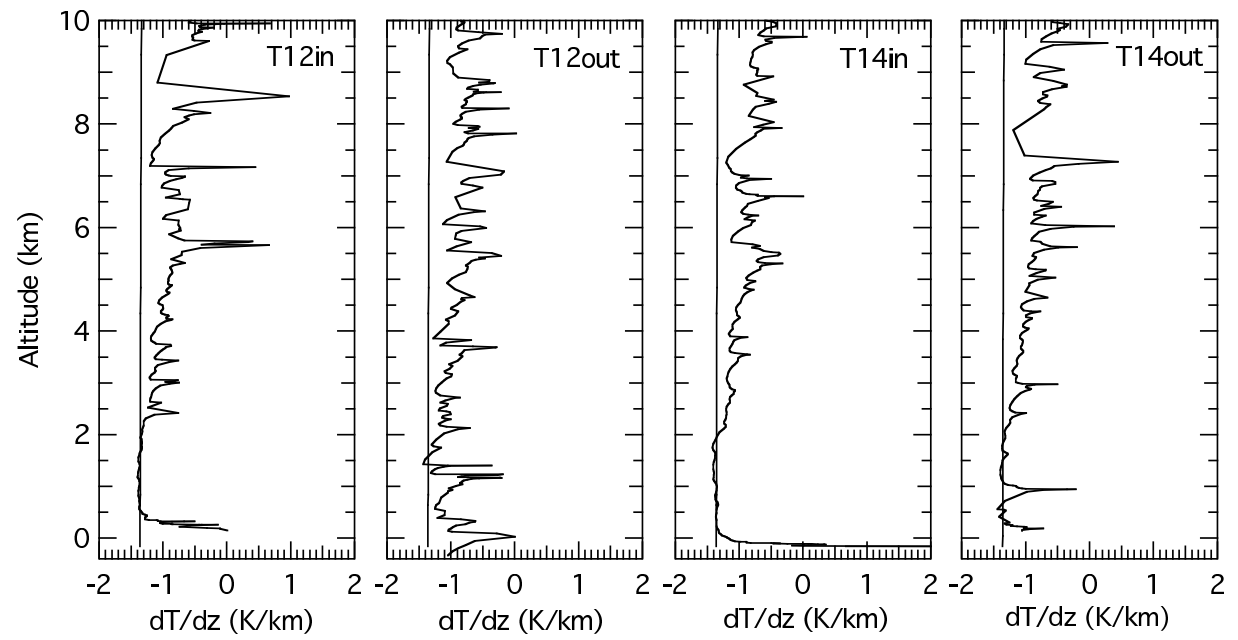

Figure 11. Vertical gradients of temperature in the lowest $10 \mathrm{~km}$ for the T12 and T14 soundings. The vertical straight line in each panel corresponds to the dry adiabat. 

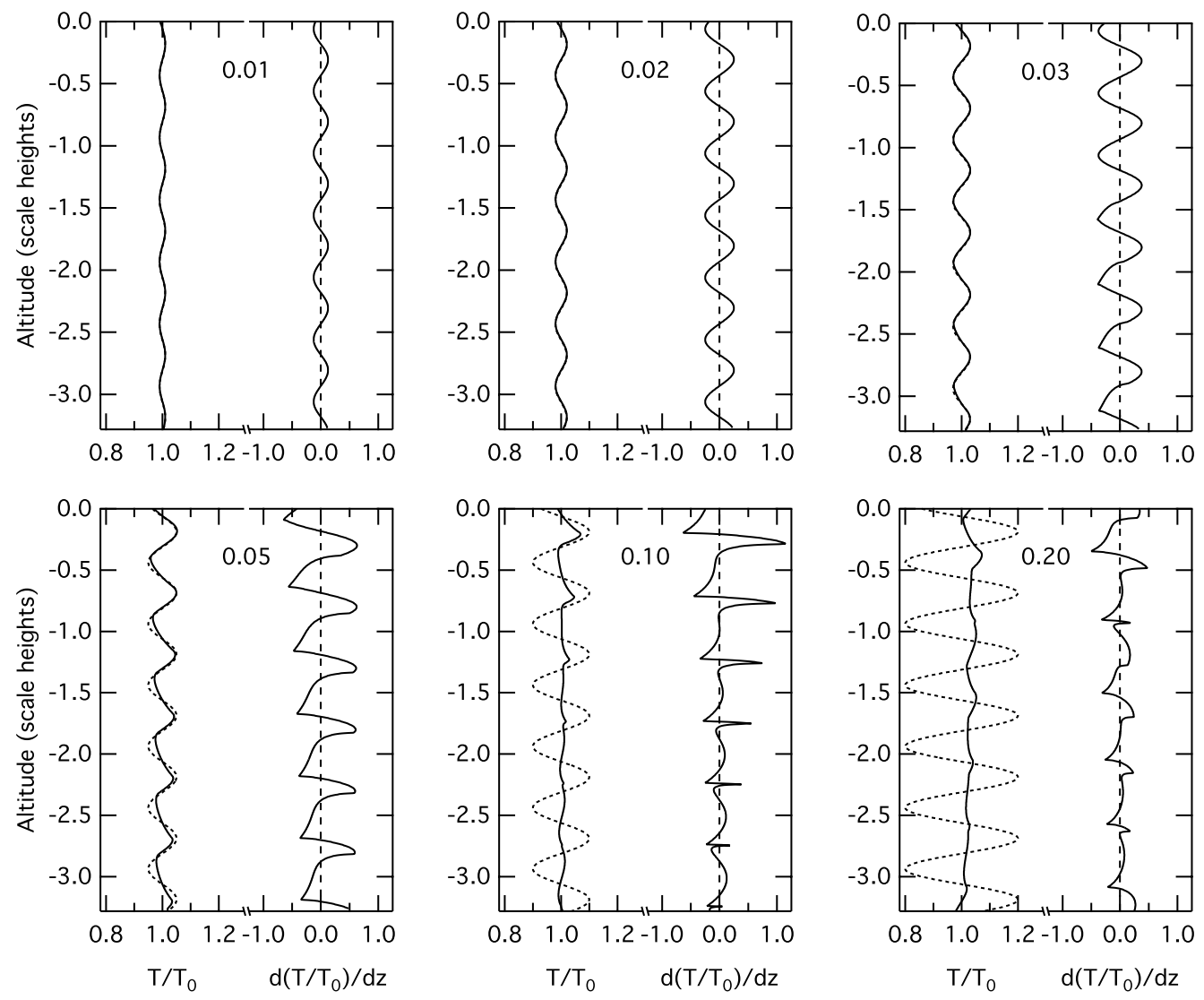

Figure 12. Retrieved temperatures and their vertical gradients from Abel inversions for an isothermal atmosphere with a sinusoidal perturbation with fractional amplitude indicated in each panel. Temperatures and gradients are normalized by the mean isothermal temperature, $T_{0}$. Dotted curves. Input perturbation temperatures, used in the forward ray-tracing calculation of light curves. Solid curves. Temperatures retrieved from the light curves using Abel inversions and the associated vertical gradients. Zero altitude corresponds to the half-power point on the light curve. The model calculations do not include a lower bounding surface. After French and Lovelace (1983). 